Review

\title{
Quantum Thermodynamics: A Dynamical Viewpoint
}

\section{Ronnie Kosloff}

Institute of Chemistry, The Hebrew University, Jerusalem 91904, Israel; E-Mail: ronnie@ @h.huji.ac.il; Tel.: + 972-26585485; Fax: + 972-26513742

Received: 26 March 2013; in revised form: 21 May 2013 /Accepted: 23 May 2013 /

Published: 29 May 2013

\begin{abstract}
Quantum thermodynamics addresses the emergence of thermodynamic laws from quantum mechanics. The viewpoint advocated is based on the intimate connection of quantum thermodynamics with the theory of open quantum systems. Quantum mechanics inserts dynamics into thermodynamics, giving a sound foundation to finite-time-thermodynamics. The emergence of the 0-law, I-law, II-law and III-law of thermodynamics from quantum considerations is presented. The emphasis is on consistency between the two theories, which address the same subject from different foundations. We claim that inconsistency is the result of faulty analysis, pointing to flaws in approximations.
\end{abstract}

Keywords: thermodynamics; open quantum systems; heat engines; refrigerators; quantum devices; finite time thermodynamics

\section{Introduction}

Quantum thermodynamics is the study of thermodynamic processes within the context of quantum dynamics. Thermodynamics preceded quantum mechanics; consistency with thermodynamics led to Planck's law, the dawn of quantum theory. Following the ideas of Planck on black body radiation, Einstein (1905) quantized the electromagnetic field [1]. Quantum thermodynamics is devoted to unraveling the intimate connection between the laws of thermodynamics and their quantum origin, requiring consistency. For many decades, the two theories developed separately. An exception is the study of Scovil et al. [2,3], which showed the equivalence of the Carnot engine [4] with the three level Maser, setting the stage for new developments.

With the establishment of quantum theory, the emergence of thermodynamics from quantum mechanics becomes a key issue. The two theories address the same subject from different viewpoints. 
This requires a consistent view of the state and dynamics of matter. Despite its name, dynamics is absent from most thermodynamic descriptions. The standard theory concentrates on systems close to equilibrium. This is also the prevailing view in quantum statistical mechanics, which is outside the scope of this thesis. Quantum mechanics has been used to reintroduce dynamical processes into thermodynamics. In particular, the theory of quantum open systems supplies the framework to separate the system from its environment. The Markovian master equation pioneered by Lindblad and Gorini-Kossakowski-Sudarshan (LGKS generator) [5,6] is one of the key elements of the theory of quantum thermodynamics $[7,8]$. The dynamical framework allows one to reinterpret and justify the theory of finite time thermodynamics [9-11], which addresses thermodynamic processes taking place in finite time.

A thermodynamic tradition is learning by example. The model of a heat engine by Carnot [4] led to generalizations; the first and second law of thermodynamics. A quantum mechanical model of heat engines and refrigerators allows us to incorporate dynamics into thermodynamics. Two types of devices have been studied: reciprocating engines utilizing the Otto and Carnot cycle and continuous engines resembling lasers and laser cooling devices. A reciprocating cycle is partitioned typically into four segments, two adiabats, where the working system is isolated from the environment, and two heat transfer segments, either isotherms for the Carnot cycle [12-17] or isochores for the Otto cycle [18-31]. The same cycles were then used as models for refrigerators [19,32-35].

In quantum thermodynamics, adiabats are modeled by time-dependent Hamiltonians. Typically, the external control Hamiltonian does not commute with the internal Hamiltonian. Infinitely slow operation is the prerequisite for the quantum and thermodynamic adiabatic conditions. Under these conditions, the engine's cycle has zero power. To generate finite power, the speed of operation has to be increased. Empirically, it is known that faster motion leads to losses, due to friction. The quantum description identifies the source of friction in the inability of the system to stay diagonal in the instantaneous energy frame [27,36-39]. Once energy is accounted for, which in an engine cycle, occurs on the heat transfer segments, the off-diagonal elements are wiped out. This loss leads to the phenomenon of friction $[27,38]$. For special cases when the dynamics can be described by a closed set of observables, the friction can be reduced or eliminated by requiring that only at the initial and final points of the adiabatic state, the systems are diagonal in energy. This phenomena has been termed "shortcut to adiabaticity" [32,40-44].

In quantum thermodynamics, the heat transfer segments are described by a system-bath interaction modeled by open quantum system techniques. The LGKS generator [5,6] is typically employed $[12,15,17,18]$. For finite power operation, the thermal transfer process is never allowed to reach equilibrium with the heat bath, which would take an infinite amount of time. Finally, maximum power output is obtained by optimizing the time allocation on each of the segments of the cycle. The efficiency of the engine at maximum power can then be compared to the well studied results of finite-time thermodynamics [9-11,45-49]. In the limit of high temperature, the quantum model converges to the finite-time thermodynamic result $[12,27,50]$.

The prime example of a continuous quantum engine is a three-level laser, which has been shown to be limited by Carnot efficiency [2,3]. Power optimization leads to efficiency at maximum power identical to finite-time thermodynamics [8]. Many dynamical models have been introduced for different types of continuous quantum engines, all consistent with the laws of thermodynamics [7,8,13,20,24,25,51-65]. 
The prime example of a continuous refrigerator is laser cooling. In this context, it is obtained by reversing the operation of a three-level laser [51,52,66-78]. A quantum absorption refrigerator has also been studied, which is a refrigerator with heat as its power source $[53,70,79,80]$. An example could be a refrigerator driven by sunlight [81].

Amazingly, in all these examples, a thermodynamic description is appropriate up to the level of a single open quantum system $[8,35,62,82]$. The common assumption that thermodynamics applies solely to macroscopic systems is only true in classical mechanics.

There are alternative approaches to the emergence of thermodynamic phenomena, for example, based on the complexity of the spectrum of large quantum systems [83-86]. For such systems, the closed system dynamics of global observables is indistinguishable from dynamics generated by LGKS generators $[87,88]$. The term quantum thermodynamics has been introduced by Partovi to describe a particle interacting with a heat reservoir and its rate of approach to equilibrium.[89].

Two contemporary fields of research—ultra cold matter and quantum information processing — are closely related to quantum thermodynamics. Cooling mechanical systems unravel their quantum character. As the temperature decreases, degrees of freedom freeze out, leaving a simplified dilute effective Hilbert space. Ultracold quantum systems contributed significantly to our understanding of basic quantum concepts. In addition, such systems form the basis for emerging quantum technologies. The necessity to reach ultra-low temperatures requires focus on the cooling process itself, i.e., quantum refrigeration. A key theme in quantum information processes is error correction. The resource for these operations are cold ancilla qubits [90]. It is therefore expected that a quantum computer will be intimately connected to a quantum refrigerator.

The present review follows the manifestation of the laws of thermodynamics in their quantum dynamical context.

- The 0-law of thermodynamics deals with the partition of the system from the bath.

- I-law: The first law of thermodynamics is a statement of conservation of energy.

- II-law: The second law is a statement on the irreversibility of dynamics: the breakup of time reversal symmetry. An empirical definition: heat will flow spontaneously from a hot source to a cold sink. These statements are translated to quantum definitions of positive entropy generation.

- III-law: We will analyze two formulations. The first is that the entropy of any pure substance in thermodynamic equilibrium approaches zero as the temperature approaches absolute zero. The second formulation is a dynamical one, known as the unattainability principle: it is impossible by any procedure, no matter how idealized, to reduce any assembly to absolute zero temperature in a finite number of operations.

\section{Quantum Open Systems}

Quantum thermodynamics is based on a series of idealizations in similar fashion to the ideal gas model, which serves classical thermodynamics. The theory of quantum open systems is the inspiration for many of these idealizations. The primary goal of open quantum systems theory is to develop a local dynamical description of the dynamics of a system coupled to an environment termed "reduced dynamics". The variables of the theory are defined by local system observables. These 
observables constitute the quantum thermodynamic description. To account for possible system-bath entanglement, the system has to be described by a mixed state, $\hat{\boldsymbol{\rho}}_{S}$. Observables are obtained from: $\langle\hat{\mathbf{O}}\rangle=\operatorname{Tr}\left\{\hat{\boldsymbol{\rho}}_{S} \hat{\mathbf{O}}\right\}[91]$.

It is customary to assume that the entire world is a large closed system, and therefore, time evolution is governed by a unitary transformation generated by a global Hamiltonian. For the combined system bath scenario, the global Hamiltonian can be decomposed into:

$$
\hat{\mathbf{H}}=\hat{\mathbf{H}}_{S}+\hat{\mathbf{H}}_{B}+\hat{\mathbf{H}}_{S B}
$$

where $\hat{\mathbf{H}}_{S}$ is the system's Hamiltonian, $\hat{\mathbf{H}}_{B}$ the bath Hamiltonian and $\hat{\mathbf{H}}_{S B}$ the system-bath interaction. Formally, the state of the system can be obtained from a partial trace over the combined system: $\hat{\rho}_{S}(t)=$ $\operatorname{Tr}_{B}\left\{\hat{\boldsymbol{\rho}}_{S B}(t)\right\}=\operatorname{Tr}_{B}\left\{\hat{\mathbf{U}} \hat{\boldsymbol{\rho}}_{S B}(0) \hat{\mathbf{U}}^{\dagger}\right\}$ where $\hat{\mathbf{U}}$ is generated by the total Hamiltonian: $\hat{\mathbf{U}}=e^{-\frac{i}{\hbar} \hat{\mathbf{H}} t}$. Reduced dynamics is an equivalent description utilizing only systems operators. The desired outcome is to obtain a local dynamical theory.

There are two major strategies to derive such equations. The first is based on the weak system-bath coupling expansion, which leads to an integro-differential equation for the systems state, $\hat{\boldsymbol{\rho}}_{S}$ [92]:

$$
\frac{d}{d t} \hat{\boldsymbol{\rho}}_{S}(t)=-\frac{i}{\hbar}\left[\hat{\mathbf{H}}_{S}, \hat{\boldsymbol{\rho}}_{S}(t)\right]+\int_{0}^{t} \mathcal{K}\left(t, t^{\prime}\right) \hat{\boldsymbol{\rho}}_{S}\left(t^{\prime}\right) d t^{\prime}
$$

where $\mathcal{K}$ is the memory kernel, and an additional assumption that at $t=0$, the system and bath are uncorrelated:

$$
\hat{\boldsymbol{\rho}}=\hat{\boldsymbol{\rho}}_{S} \otimes \hat{\boldsymbol{\rho}}_{B}
$$

Assuming the bath dynamics is fast, Equation (2) is reduced to a differential form [93,94]:

$$
\frac{d}{d t} \hat{\boldsymbol{\rho}}_{S}=-\frac{i}{\hbar}\left[\hat{\mathbf{H}}_{S}, \hat{\boldsymbol{\rho}}_{S}\right]+\mathcal{L}_{D} \hat{\boldsymbol{\rho}}_{S}
$$

where $\mathcal{L}_{D}$ is the generator of dissipative dynamics.

The alternative approach to reduced dynamics searches for the most general form of the generator of Markovian dynamics or, in more technical terms, a completely positive quantum dynamical semigroup [92,95]. Kraus has shown [96] that starting from an uncorrelated initial system and bath state in Equation (3), a reduced map, $\Lambda_{S}(t)$, to the final time always has the structure:

$$
\hat{\boldsymbol{\rho}}_{S}(t)=\Lambda_{S}(t) \hat{\boldsymbol{\rho}}_{S}(0)=\sum_{j} \hat{\mathbf{K}}_{j}(t) \hat{\boldsymbol{\rho}}_{S}(0) \hat{\mathbf{K}}_{j}^{\dagger}(t)
$$

where $\hat{\mathbf{K}}$ are system operators and $\sum_{j} \hat{\mathbf{K}}_{j} \hat{\mathbf{K}}_{j}^{\dagger}=\hat{\mathbf{I}}$. When adding a Markovian assumption, $\Lambda_{S}(t)=e^{\mathcal{L} t}$, the differential generator, $\mathcal{L}$, of the map becomes $[5,6]$ :

$$
\frac{d}{d t} \hat{\boldsymbol{\rho}}_{S}=\mathcal{L} \hat{\boldsymbol{\rho}}_{S}=-\frac{i}{\hbar}\left[\hat{\mathbf{H}}_{S}, \hat{\boldsymbol{\rho}}_{S}\right]+\sum_{j}\left(\hat{\mathbf{V}}_{j} \hat{\boldsymbol{\rho}}_{S} \hat{\mathbf{V}}_{j}^{\dagger}-\frac{1}{2}\left\{\hat{\mathbf{V}}_{j}^{\dagger} \hat{\mathbf{V}}_{j}, \hat{\boldsymbol{\rho}}_{S}\right\}\right)
$$

where $\hat{\mathbf{V}}$ are system operators and $\hat{\mathbf{H}}_{S}$ is a renormalized system Hamiltonian. Equation (6) is the Lindblad-Gorini-Kossakowski-Sudarshan (LGKS)-semi-group generator [5,6]. In the LGKS derivation, the operators, $\hat{\mathbf{V}}_{j}$, are unrestricted system operators. Explicit derivations, such as in Equation (23), 
relate the operators, $\hat{\mathbf{V}}_{j}$, to system-bath coupling terms. The Markovian dynamics implies also that Equation (3) is true for all times [97]. The completely positive construction assures that the dynamics can be generated from a non-unique Hamiltonian model of some large system.

The Heisenberg representation supplies a direct link to quantum thermodynamic observables. The dynamics of an observable represented by the operator, $\hat{O}$, has the form:

$$
\frac{d}{d t} \hat{\mathbf{O}}=\mathcal{L}^{*} \hat{\mathbf{O}}=+\frac{i}{\hbar}\left[\hat{\mathbf{H}}_{S}, \hat{\mathbf{O}}\right]+\sum_{j}\left(\hat{\mathbf{V}}_{j} \hat{\mathbf{O}} \hat{\mathbf{V}}_{j}^{\dagger}-\frac{1}{2}\left\{\hat{\mathbf{V}}_{j} \hat{\mathbf{V}}_{j}^{\dagger}, \hat{\mathbf{O}}\right\}\right)+\frac{\partial \hat{\mathbf{O}}}{\partial t}
$$

where the possibility that the operator, Ô, which is explicitly time-dependent, is included. Equation (7) allows one to follow, in time, thermodynamic observables, such as energy, for a desired process.

\section{The 0-Law}

The zero law of thermodynamics is typically stated as: If $A$ and $C$ are each in thermal equilibrium with $B, A$ is also in equilibrium with $C$. A thermodynamic description is based on idealized partitions between subsystems. An isothermal partition, for example, allows heat to flow from the system to the bath, maintaining the integrity of the subsystems. Consistency with quantum mechanics due to the global structure of the theory is therefore a non-trivial statement.

\subsection{System Bath Partition}

Quantum thermodynamics idealizes that the system can be fully described by local operators, which is equivalent to the condition:

$$
\hat{\boldsymbol{\rho}} \approx \hat{\boldsymbol{\rho}}_{S} \otimes \hat{\boldsymbol{\rho}}_{B}
$$

In Equation (8), there is no system-bath entanglement, which is also true for Markovian dynamics. Thermodynamically, the local description of the system is equivalent to the extensivity of its observables. We conclude that the dynamics represented by the LGKS generator in Equation (6) is closely linked to a thermodynamic framework.

\subsection{Thermal Equilibrium}

An equilibrium state, in general, is defined as stationary and stable. This assumption is used to derive the Kubo-Martin-Schwinger stability criterion for thermal equilibrium $[98,99]$. This criterion will imply that in equilibrium, there is no energy current between the system and bath. If we extend the description to a network of baths connected by systems:

$$
\hat{\mathbf{H}}=\hat{\mathbf{H}}_{S_{1}}+\hat{\mathbf{H}}_{B_{a}}+\hat{\mathbf{H}}_{S_{1} B_{a}}+\hat{\mathbf{H}}_{S_{2}}+\hat{\mathbf{H}}_{S_{2} B_{b}}+\hat{\mathbf{H}}_{B_{b}}+\hat{\mathbf{H}}_{S_{1} B_{b}}+\hat{\mathbf{H}}_{S_{2} B_{a}} \ldots
$$

then the KMS condition for a tensor product state of subsystems implies the 0-law for a network of coupled systems. 


\section{The I-Law}

The I-law is devoted to the elusive concept of energy conservation. If the universe is closed and passive, meaning there are no source terms, then its energy is conserved. This implies that the total evolution is unitary, where the dynamics are generated by a total Hamiltonian, $\hat{\mathbf{H}}$, cf. Equations (1) and (9). As a result, the total energy expectation, $\langle\hat{\mathbf{H}}\rangle$, is constant.

Quantum thermodynamics focuses on the balance of energy of systems coupled to a bath. The local change is the sum of the heat currents in and out of the system: heat flow from the environments and power from an external source. The time derivative of the system's energy balance becomes:

$$
\frac{d E_{S}}{d t}=\sum_{j}^{N} \mathcal{J}_{j}+\mathcal{P}
$$

where $\mathcal{J}_{j}$ is the heat current from the $j$ th bath and $\mathcal{P}$ is the external power. The quantum thermodynamic version of the I-law is obtained by inserting the system Hamiltonian, $\hat{\mathbf{H}}_{S}$, into Equation (7), leading to:

$$
\frac{d E_{S}}{d t}=\left\langle\frac{\partial \hat{\mathbf{H}}_{S}}{\partial t}\right\rangle+\left\langle\mathcal{L}_{D}\left(\hat{\mathbf{H}}_{S}\right)\right\rangle=\left\langle\frac{\partial \hat{\mathbf{H}}_{S}}{\partial t}\right\rangle+\sum_{j}^{N} \mathcal{J}_{j}
$$

Since $\left[\hat{\mathbf{H}}_{S}, \hat{\mathbf{H}}_{S}\right]=0$, only the dissipative part of $\mathcal{L}$ appears. The heat currents, $\mathcal{J}_{j}$, can be identified as:

$$
\mathcal{J}_{j}=\left\langle\hat{\mathbf{V}}_{j} \hat{\mathbf{H}}_{S} \hat{\mathbf{V}}_{j}^{\dagger}-\frac{1}{2}\left\{\hat{\mathbf{V}}_{j} \hat{\mathbf{V}}_{j}^{\dagger}, \hat{\mathbf{H}}_{S}\right\}\right\rangle
$$

and the power becomes:

$$
\mathcal{P}=\left\langle\frac{\partial H_{S}}{\partial t}\right\rangle
$$

Equations (12) and (13) are the dynamical versions of the I-law based on Markovian dynamics $[8,100,101]$.

The criticism of relying exclusively on LGKS generators is that they are not unique. In addition, such reliance may violate the II-law. The non-uniqueness is caused by the substitution, $\hat{\mathbf{V}} \rightarrow \hat{\mathbf{V}}+i \gamma \hat{\mathbf{I}}$ and $\hat{\mathbf{H}}_{S} \rightarrow \hat{\mathbf{H}}_{S}+\frac{\gamma}{2}\left(\hat{\mathbf{V}}+\hat{\mathbf{V}}^{\dagger}\right)$, which maintains the dynamics generated by the Equation (7) invariant. In contradiction, the heat current, $\mathcal{L}_{D}\left(\hat{\mathbf{H}}_{S}\right)$, is not invariant to this transformation. The origin of this fuzziness of the definition stems from the arbitrariness of accounting for the system bath interaction energy in $\hat{\mathbf{H}}_{S}$. To obtain a consistent definition of the I-law, additional restrictions must be imposed.

\subsection{The Dynamical Generator in the Weak System-Bath Coupling Limit}

A unique and consistent approach is obtained by deriving the generator, $\mathcal{L}_{D}$, in the weak systembath coupling limit. In this limit, the interaction energy can be neglected. This approach represents a thermodynamic idealization: it allowes energy transfer, while keeping a tensor product separation between the system and bath, i.e., a quantum version of an isothermal partition.

Consider a system and a reservoir (bath), with a "bare" system Hamiltonian, $\hat{\mathbf{H}}_{S}$, and the bath Hamiltonian, $\hat{\mathbf{H}}_{B}$, interacting via the Hamiltonian, $\lambda \hat{\mathbf{H}}_{\text {int }}=\lambda \hat{\mathbf{S}} \otimes \hat{\mathbf{B}}$. Here, $\lambda$ is the coupling strength. It is assumed that the bath is stationary:

$$
\left[\hat{\boldsymbol{\rho}}_{B}, \hat{\mathbf{H}}_{B}\right]=0, \operatorname{Tr}\left\{\hat{\boldsymbol{\rho}}_{B} \hat{\mathbf{B}}\right\}=0
$$


The reduced, system-only dynamics in the interaction picture map are defined by a partial trace over the bath:

$$
\hat{\boldsymbol{\rho}}_{S}(t)=\Lambda(t, 0) \hat{\boldsymbol{\rho}}_{S} \equiv \operatorname{Tr}_{B}\left\{\hat{\mathbf{U}}_{\lambda}(t, 0) \hat{\boldsymbol{\rho}}_{S} \otimes \hat{\boldsymbol{\rho}}_{B} \hat{\mathbf{U}}_{\lambda}(t, 0)^{\dagger}\right\}
$$

where the unitary propagator in the interaction picture is described by the time-ordered exponential:

$$
\hat{\mathbf{U}}_{\lambda}(t, 0)=\mathcal{T} \exp \left\{\frac{-i \lambda}{\hbar} \int_{0}^{t} \hat{\mathbf{S}}(s) \otimes \hat{\mathbf{B}}(s) d s\right\}
$$

where:

$$
\hat{\mathbf{S}}(t)=e^{(i / \hbar)} \hat{\mathbf{H}}_{S}^{\prime} t \hat{\mathbf{S}} e^{-(i / \hbar) \hat{\mathbf{H}}_{S}^{\prime} t}, \hat{\mathbf{B}}(t)=e^{(i / \hbar) \hat{\mathbf{H}}_{B} t} \hat{\mathbf{B}} e^{-(i / \hbar) \hat{\mathbf{H}}_{B} t}
$$

$\hat{\mathbf{S}}(t)$ is defined with respect to the renormalized, physical $\hat{\mathbf{H}}_{S}^{\prime}$ and not $\hat{\mathbf{H}}_{S}$, which can be expressed as:

$$
\hat{\mathbf{H}}_{S}^{\prime}=\hat{\mathbf{H}}_{S}+\lambda^{2} \hat{\mathbf{H}}_{1}^{\mathrm{corr}}+\cdots
$$

The re-normalizing terms containing powers of $\lambda$ are Lamb-shift corrections due to the interaction with the bath. The lowest order (Born) approximation with respect to the coupling constant, $\lambda$, yields $\hat{\mathbf{H}}_{1}^{\text {corr }}$.

A convenient tool to represent the reduced map is a cumulant expansion:

$$
\hat{\Lambda}(t, 0)=\exp \sum_{n=1}^{\infty}\left[\lambda^{n} \mathcal{K}^{(n)}(t)\right]
$$

One finds that $\mathcal{K}^{(1)}=0$ and the weak coupling limit consist of terminating the cumulant expansion at $n=2$; hence, we denote the generator, $\mathcal{K}^{(2)} \equiv \mathcal{K}$ :

$$
\hat{\mathbf{\Lambda}}(t, 0)=\exp \left[\lambda^{2} \mathcal{K}(t)+\hat{\mathbf{O}}\left(\lambda^{3}\right)\right]
$$

One obtains:

$$
\mathcal{K}(t) \hat{\boldsymbol{\rho}}_{S}=\frac{1}{\hbar^{2}} \int_{0}^{t} d s \int_{0}^{t} d u F(s-u) \hat{\mathbf{S}}(s) \hat{\boldsymbol{\rho}}_{S} \hat{\mathbf{S}}(u)^{\dagger}+(\text { similar terms })
$$

where $F(s)=\operatorname{Tr}\left\{\hat{\boldsymbol{\rho}}_{B} \hat{\mathbf{B}}(s) \hat{\mathbf{B}}\right\}$. The similar terms in Equation (21) are of the form $\hat{\boldsymbol{\rho}}_{S} \hat{\mathbf{S}}(s) \hat{\mathbf{S}}(u)^{\dagger}$ and $\hat{\mathbf{S}}(s) \hat{\mathbf{S}}(u)^{\dagger} \hat{\boldsymbol{\rho}}_{S}$.

The Markov approximation (in the interaction picture) means that for a sufficiently long time, the generator in Equation (19) becomes:

$$
\mathcal{K}(t) \simeq t \mathcal{L}
$$

where $\mathcal{L}$ is a Linblad-Gorini-Kossakowski-Sudarshan (LGKS) generator [5,6]. To find its form, the effective system coupling term, $\hat{\mathbf{S}}(t)$, is decomposed into its Fourier components:

$$
\hat{\mathbf{S}}(t)=\sum_{\{\omega\}} e^{i \omega t} \hat{\mathbf{S}}_{\omega}, \quad \hat{\mathbf{S}}_{-\omega}=\hat{\mathbf{S}}_{\omega}^{\dagger}
$$

where the set, $\{\omega\}$, contains Bohr frequencies $\omega=\epsilon_{k}-\epsilon_{l}$ of the Hamiltonian:

$$
\hat{\mathbf{H}}_{S}^{\prime}=\sum_{k} \epsilon_{k}|k\rangle\langle k|
$$


Then, Equation (21) can be written as:

$$
\mathcal{K}(t) \hat{\boldsymbol{\rho}}_{S}=\frac{1}{\hbar^{2}} \sum_{\omega, \omega^{\prime}} \hat{\mathbf{S}}_{\omega} \hat{\boldsymbol{\rho}}_{S} \hat{\mathbf{S}}_{\omega^{\prime}}^{\dagger} \int_{0}^{t} e^{i\left(\omega-\omega^{\prime}\right) u} d u \int_{-u}^{t-u} F(\tau) e^{i \omega \tau} d \tau+(\text { similar terms })
$$

with the use of two crucial approximations:

$$
\int_{0}^{t} e^{i\left(\omega-\omega^{\prime}\right) u} d u \approx t \delta_{\omega \omega^{\prime}}, \quad \int_{-u}^{t-u} F(\tau) e^{i \omega \tau} d \tau \approx G(\omega)=\int_{-\infty}^{\infty} F(\tau) e^{i \omega \tau} d \tau \geq 0
$$

This condition works for $t \gg \max \left\{1 /\left(\omega-\omega^{\prime}\right)\right\}$. These two approximations lead to $\mathcal{K}(t) \hat{\boldsymbol{\rho}}_{S}=$ $\left(t / \hbar^{2}\right) \sum_{\omega} \hat{\mathbf{S}}_{\omega} \hat{\boldsymbol{\rho}}_{S} \hat{\mathbf{S}}_{\omega}^{\dagger} G(\omega)+\left(\right.$ similar terms), and hence, it follows from Equation (22) that $\mathcal{L}_{D}$ is a special case of the LGKS generator [ Equation (6) ] derived for the first time by Davies [93]. Returning to the Schrödinger picture, one obtains the following Markovian master equation:

$$
\begin{aligned}
\frac{d \hat{\boldsymbol{\rho}}_{S}}{d t} & =-\frac{i}{\hbar}\left[\hat{\mathbf{H}}_{S}^{\prime}, \hat{\boldsymbol{\rho}}_{S}\right]+\mathcal{L}_{D} \hat{\boldsymbol{\rho}}_{S} \\
\mathcal{L}_{D} \hat{\boldsymbol{\rho}}_{S} & \equiv \frac{\lambda^{2}}{2 \hbar^{2}} \sum_{\{\omega\}} G(\omega)\left(\left[\hat{\mathbf{S}}_{\omega}, \hat{\boldsymbol{\rho}}_{S} \hat{\mathbf{S}}_{\omega}^{\dagger}\right]+\left[\hat{\mathbf{S}}_{\omega} \hat{\boldsymbol{\rho}}_{S}, \hat{\mathbf{S}}_{\omega}^{\dagger}\right]\right)
\end{aligned}
$$

The positivity, $G(\omega) \geq 0$, follows from Bochner's theorem and is a necessary condition for the complete positivity of the Markovian master equation.

The absence of off-diagonal terms in Equation (28), compared to Equation (25), is the crucial property of the Davies generator, which can be interpreted as coarse-graining in time of fast oscillating terms. It implies also the commutation of $\mathcal{L}_{D}$ with the Hamiltonian part, $\left[\hat{\mathbf{H}}_{S}^{\prime}, \bullet\right.$. This fixes the ambiguity in Equation (7) of the partition between the Hamiltonian and dissipative parts. Markovian behavior involves a rather complicated cooperation between system and bath dynamics. This means that in phenomenological treatments, one cannot combine arbitrary system Hamiltonians, $\hat{\mathbf{H}}_{S}$, with a given LGKS generator. This observation is particularly important in the context of quantum thermodynamics, where it is tempting to study Markovian dynamics with an arbitrary control Hamiltonian. Erroneous derivations of the quantum master equation can easily lead to a violation of the laws of thermodynamics, cf. next section.

For a bath at thermal equilibrium, the additional Kubo-Martin-Schwinger (KMS) [98,99] condition holds:

$$
G(-\omega)=\exp \left(-\frac{\hbar \omega}{k_{B} T}\right) G(\omega)
$$

where $T$ is the bath's temperature. As a consequence of Equation (29), the Gibbs state:

$$
\hat{\boldsymbol{\rho}}_{\beta}=Z^{-1} e^{-\beta \hat{\mathbf{H}}_{S}^{\prime}}, \beta=\frac{1}{k_{B} T}
$$

is a stationary solution of Equation (28). Under mild conditions (e.g., the only system operators commuting with $\hat{\mathbf{H}}_{S}^{\prime}$ and $\hat{\mathbf{S}}$ are scalars), the Gibbs state is a unique stationary state, and any initial state relaxes towards equilibrium, which is consistent with the 0 -th law of thermodynamics. The corresponding thermal generator in Heisenberg form becomes:

$$
\mathcal{L}_{D}^{*} \hat{\mathbf{O}}=\sum_{\{\omega \geq 0\}} \gamma(\omega)\left(\left(\hat{\mathbf{S}}_{\omega}, \hat{\mathbf{O}} \hat{\mathbf{S}}_{\omega}^{\dagger}-\frac{1}{2}\left\{\hat{\mathbf{S}}_{\omega} \hat{\mathbf{S}}_{\omega}^{\dagger}, \hat{\mathbf{O}}\right\}\right)+e^{-\hbar \beta \omega}\left(\hat{\mathbf{S}}_{\omega}^{\dagger}, \hat{\mathbf{O}} \hat{\mathbf{S}}_{\omega}-\frac{1}{2}\left\{\hat{\mathbf{S}}_{\omega}^{\dagger} \hat{\mathbf{S}}_{\omega}, \hat{\mathbf{O}}\right\}\right)\right)
$$


where, finally, the kinetic coefficients become Fourier transforms of the bath autocorrelation functions:

$$
\gamma(\omega)=\frac{\lambda^{2}}{\hbar^{2}} \int_{-\infty}^{+\infty} \operatorname{Tr}\left\{\hat{\boldsymbol{\rho}}_{B} e^{i \hat{\mathbf{H}}_{B} t / \hbar} \hat{\mathbf{B}} e^{-i \hat{\mathbf{H}}_{B} t / \hbar} \hat{\mathbf{B}}\right\} e^{-i \omega t} d t
$$

The weak system bath coupling is the quantum version of the thermodynamic isothermal partition between the system and bath. It preserves the autonomy of the system's observables, allowing energy flow across the boundary, thus restoring the definition of the heat flow, $\mathcal{J}$, in Equation (12) and power in Equation (13).

\subsection{Thermal Generators for Periodic Driving Fields}

An external perturbation modifying the Hamiltonian of the system will also modify the heat flow. As a result, the LGKS generator has to be renormalized. For a slow change, one can adopt the adiabatic approach and use the instantaneous system's Hamiltonian to derive $\mathcal{L}_{D}$. In general, the temporal changes in the system's Hamiltonian have to be incorporated in the derivation of the dissipative generator. This task is difficult, since fast changes may alter the Markovian assumption. An important class of problems in quantum thermodynamics is periodically driven systems. Periodic heat engines and power-driven refrigerators fall into this class.

A derivation of the dissipative generator, $\mathcal{L}_{D}$, limited to a periodically driven time-dependent Hamiltonian, [ cf. Equation (13)], is sketched.

(1) The system's renormalized Hamiltonian is now periodic in time:

$$
\hat{\mathbf{H}}_{S}(t)=\hat{\mathbf{H}}_{S}(t+\tau), \hat{\mathbf{U}}(t, 0) \equiv \mathcal{T} \exp \left\{-\frac{i}{\hbar} \int_{0}^{t} \hat{\mathbf{H}}_{S}(s) d s\right\}
$$

The role of the constant Hamiltonian is played by an effective Hamiltonian $\hat{\mathbf{H}}_{e f f}$ defined by the spectrum of the periodic propagator:

$$
\hat{\mathbf{H}}_{e f f}=\sum_{k} \epsilon_{k}|k\rangle\langle k|, \hat{\mathbf{U}}(\tau, 0)=e^{-i \frac{1}{\hbar} \hat{\mathbf{H}}_{e f f} \tau}
$$

the $\epsilon_{k}$ are called "quasi-energies".

(2) The Fourier decomposition (23) is replaced by a double Fourier decomposition:

$$
\hat{\mathbf{U}}(t, 0)^{\dagger} \hat{\mathbf{S}} \hat{\mathbf{U}}(t, 0)=\sum_{q \in \mathbf{Z}} \sum_{\{\omega\}} e^{i(\omega+q \Omega) t} \hat{\mathbf{S}}_{\omega q}
$$

where $\Omega=2 \pi / \tau$ and $\{\omega\}=\left\{\epsilon_{k}-\epsilon_{l}\right\}$. The decomposition above follows from Floquet theory.

(3) The generator in the interaction picture is the sum of its Fourier components:

$$
\mathcal{L}=\sum_{q \in \mathbf{Z}} \sum_{\{\omega\}}=\mathcal{L}_{\omega q}
$$

where:

$$
\mathcal{L}_{\omega q} \hat{\boldsymbol{\rho}}_{S}=\frac{1}{2} \gamma(\omega+q \Omega)\left\{\left(\left[\hat{\mathbf{S}}_{\omega q}, \hat{\boldsymbol{\rho}}_{S} \hat{\mathbf{S}}_{\omega q}^{\dagger}\right]+\left[\hat{\mathbf{S}}_{\omega q} \hat{\boldsymbol{\rho}}_{S}, \hat{\mathbf{S}}_{\omega q}^{\dagger}\right]\right)+e^{-\hbar \beta(\omega+q \Omega)}\left(\left[\hat{\mathbf{S}}_{\omega q}^{\dagger}, \hat{\boldsymbol{\rho}}_{S} \hat{\mathbf{S}}_{\omega q}\right]+\left[\hat{\mathbf{S}}_{\omega q}^{\dagger} \hat{\boldsymbol{\rho}}_{S}, \hat{\mathbf{S}}_{\omega q}\right]\right)\right\}
$$


Returning to the Schrödinger picture, we obtain the following master equation:

$$
\frac{d \hat{\boldsymbol{\rho}}_{S}(t)}{d t}=-\frac{i}{\hbar}\left[\hat{\mathbf{H}}_{S}(t), \hat{\boldsymbol{\rho}}_{S}(t)\right]+\mathcal{L}_{D}(t) \hat{\boldsymbol{\rho}}_{S}(t), \quad t \geq 0
$$

where:

$$
\mathcal{L}(t)=\mathcal{L}(t+\tau)=\mathcal{U}(t, 0) \mathcal{L U}(t, 0)^{\dagger}, \mathcal{U}(t, 0) \cdot=U(t, 0) \cdot U(t, 0)^{\dagger}
$$

In particular, one can represent the solution of Equation (38) in the form:

$$
\rho(t)=\mathcal{U}(t, 0) e^{\mathcal{L} t} \rho(0), \quad t \geq 0
$$

Any state, satisfying $\mathcal{L} \tilde{\rho}=0$, defines a periodic steady state (limit cycle):

$$
\tilde{\rho}(t)=\mathcal{U}(t, 0) \tilde{\rho}=\tilde{\rho}(t+\tau), \quad t \geq 0
$$

Finally, one should notice that in the case of multiple couplings and multiple heat baths, the generator, $\mathcal{L}$, can be always represented as an appropriate sum of the terms, such as Equation (31).

\subsection{Heat Flows and Power for Periodically Driven Open Systems}

The heat currents for periodic systems break up to a sum of Fourier components for each bath labeled by index, $j$. Then, the generator in the interaction picture becomes:

$$
\mathcal{L}=\sum_{j=1}^{M} \sum_{q \in \mathbf{Z}} \sum_{\{\omega \geq 0\}} \mathcal{L}_{\omega q}^{j}
$$

where any single $\mathcal{L}_{\omega q}^{j}$ has a structure of Equation (37) with the appropriate $\gamma_{j}(\omega)$. The corresponding time-dependent objects satisfy:

$$
\mathcal{L}_{q \omega}^{j}(t) \tilde{\rho}_{q \omega}^{j}(t)=0, \mathcal{L}_{q \omega}^{j}(t)=\mathcal{U}(t, 0) \mathcal{L}_{q \omega}^{j} \mathcal{U}(t, 0)^{\dagger}, \tilde{\rho}_{q \omega}^{j}(t)=\mathcal{U}(t, 0) \tilde{\rho}_{q \omega}^{j}=\tilde{\rho}_{q \omega}^{j}(t+\tau)
$$

Using the decomposition in Equation (42), one can define a local heat current, which corresponds to the exchange of energy, $\omega+q \Omega$, with the $j$-th heat bath for any initial state:

$$
\mathcal{J}_{q \omega}^{j}(t)=\frac{\omega+q \Omega}{\omega} \operatorname{Tr}\left\{\left(\mathcal{L}_{q \omega}^{j}(t) \rho(t)\right) \tilde{\mathbf{H}}_{S}(t)\right\}, \tilde{\mathbf{H}}_{S}(t)=\mathcal{U}(t, 0) \hat{\mathbf{H}}_{S}
$$

The heat current associated with the $j$-th bath is a sum of the corresponding local ones:

$$
\mathcal{J}^{j}(t)=\sum_{q \in \mathbf{Z}} \sum_{\{\omega \geq 0\}} \mathcal{J}_{q \omega}^{j}(t)
$$

where the sum is over multiples of Floquet frequency and Bohr frequencies of the effective Hamiltonian.

We emphasize in this section a dynamical version of the I-law based on examining energy currents between the system and baths. This derivation for a periodically driven system is also consistent with the II-law of thermodynamics [51,80,102]. 


\section{The II-Law}

The second law is a statement on the irreversibility of dynamics or, alternatively, the breakup of time reversal symmetry. This should be consistent with the empirical direct definition: heat will flow spontaneously from a hot source to a cold sink. In classical thermodynamics, this statement is equivalent to the statement that the change in entropy of the universe is positive: $\Delta \mathcal{S} \geq 0$. In addition, the entropy generation is additive. There is considerable confusion in the adaptation of the second-law in quantum mechanics, where static and dynamical viewpoints are employed.

From a static viewpoint, for a closed quantum system, the II-law of thermodynamics is a consequence of the unitary evolution [103]. In this approach, one accounts for the entropy change before and after a change in the entire system. A dynamical viewpoint is based on local accounting for the entropy changes in the subsystems and the entropy generated in the baths.

\subsection{Entropy}

In thermodynamics, entropy is related to a concrete process. In quantum mechanics, this translates to the ability to measure and manipulate the system based on the information gathered by measurement [104]. An example is the case of Maxwell's demon, which has been resolved by Szilard [105-108]. There is a close relationship between the theory of quantum measurement and filtering out an outcome [91,109]. Gathered information can be employed to extract work from a single bath [54]. This means that the gathered information depends on the particular measurement; therefore, for the same system, different entropies appear, depending on the observable being measured. The entropy of an observable is associated with the complete projective measurement of an observable, $\hat{\mathbf{A}}$, where the operator, $\hat{\mathbf{A}}$, has a spectral decomposition: $\hat{\mathbf{A}}=\sum_{i} \alpha_{i} \hat{\mathbf{P}}_{i}$ and $\hat{\mathbf{P}}_{i}=\left|\alpha_{i}\right\rangle\left\langle\alpha_{i}\right|$. The probability of the outcome is, therefore, $p_{i}=\operatorname{tr}\left\{\hat{\boldsymbol{\rho}} \hat{\mathbf{P}}_{i}\right\}$. The entropy associated with the observable, $\hat{\mathbf{A}}$, is the Shannon entropy with respect to the possible outcomes [37]:

$$
\mathcal{S}_{A}=-\sum_{i} p_{i} \ln p_{i}
$$

where dimensionless units are chosen for entropy i.e., $k_{B}=1$. The most significant observable in thermodynamics is the energy represented by the Hamiltonian operator, $\hat{\mathbf{H}}$, and its associated energy entropy, $\mathcal{S}_{E}$ [110].

von Neumann suggested to single out the most informative observable to characterize the entropy of the system. This invariant is obtained by minimizing the entropy with respect to all possible observables. The most informative observable operator commutes with the state of the system, $[\hat{\mathbf{V}}, \hat{\boldsymbol{\rho}}]=0$. The entropy of this observable is termed the von Neumann entropy [91] and is equal to:

$$
\mathcal{S}_{v n}=-\operatorname{tr}\{\hat{\boldsymbol{\rho}} \ln \hat{\boldsymbol{\rho}}\}
$$

As a consequence, $\mathcal{S}_{A} \geq \mathcal{S}_{v n}$ for all observables, $\hat{\mathbf{A}} \neq \hat{\mathbf{V}}$. $\mathcal{S}_{v n}$ is invariant to a unitary transformation changing the state, $\hat{\rho}$. The invariance is a consequence of the entropy being a function of the eigenvalues of $\hat{\rho}$. A unitary transformation does not change these eigenvalues.

The von Neumann entropy $\mathcal{S}_{v n}$ is additive only for a system state that is composed of a tensor product of its subsystems, $\hat{\boldsymbol{\rho}}=\prod_{j} \otimes \hat{\boldsymbol{\rho}}_{j}$. In the general case, the subsystems are entangled. If local measurements 
are only accessible, then the observable entropy relates to a product of local observables, $\hat{\mathbf{O}}=\prod_{j} \otimes \hat{\mathbf{O}}_{j}$. Since this is a restricted class of operators, the associated entropy, which is the sum of the entropies of the subdivision, is always larger than the total von Neumann entropy, $\sum_{j} \mathcal{S}_{v n j} \geq \mathcal{S}_{v n}$. The extreme example is an entangled pure state, where for the bipartite case: $\mathcal{S}_{v n}=0$ and $\mathcal{S}_{v n 1}=\mathcal{S}_{v n 2}>0$. This observation is the base for using the reduced state entropy as a measure of entanglement [111-113]. Consider an uncorrelated initial state: once an interaction Hamiltonian turns on, the dynamics will cause the sum of local entropies to increase [114]. A local structure imposed on the total system by thermodynamic partitions is the source of local entropy increase. In general, entropy in quantum mechanics is not additive. Once a tensor product partition is imposed, the quantum entropy becomes additive. Alternative sources for entropy increase have been suggested. For example, in a scattering event, the correlation generated by the interaction is lost when the scattering partners reach indefinite distance [39]. Quantum complexity can also lead to quantum thermalization [85,86] and entropy increase [110].

\subsection{Quantum Networks and Quantum Devices}

A quantum network is defined as a collection of interconnected quantum systems and baths at different temperatures, cf. Figures 1 and 2. This network can be decomposed into two elementary segments: a wire and a junction. The wire is a transport line between two segments. In the most simple form, it connects two baths. The junction is a tricycle; a system combining three currents. A heat engine is a tricycle connected to three baths; a work bath and a hot and cold bath. More complex networks can be constructed from these two basic elements, cf. Figure 2. A linear network composed of linearly coupled harmonic oscillators can be decomposed to wires only [115].

Figure 1. The tricycle on the left and the wire on the right; elementary components in a quantum network. The tricycle combines three energy currents. The tricycle in the figure is connected to three heat baths, demonstrating a heat-driven refrigerator. The wire combines two energy currents. The wire in the figure is connected to a hot and cold bath. The I-law and II-law are indicated.

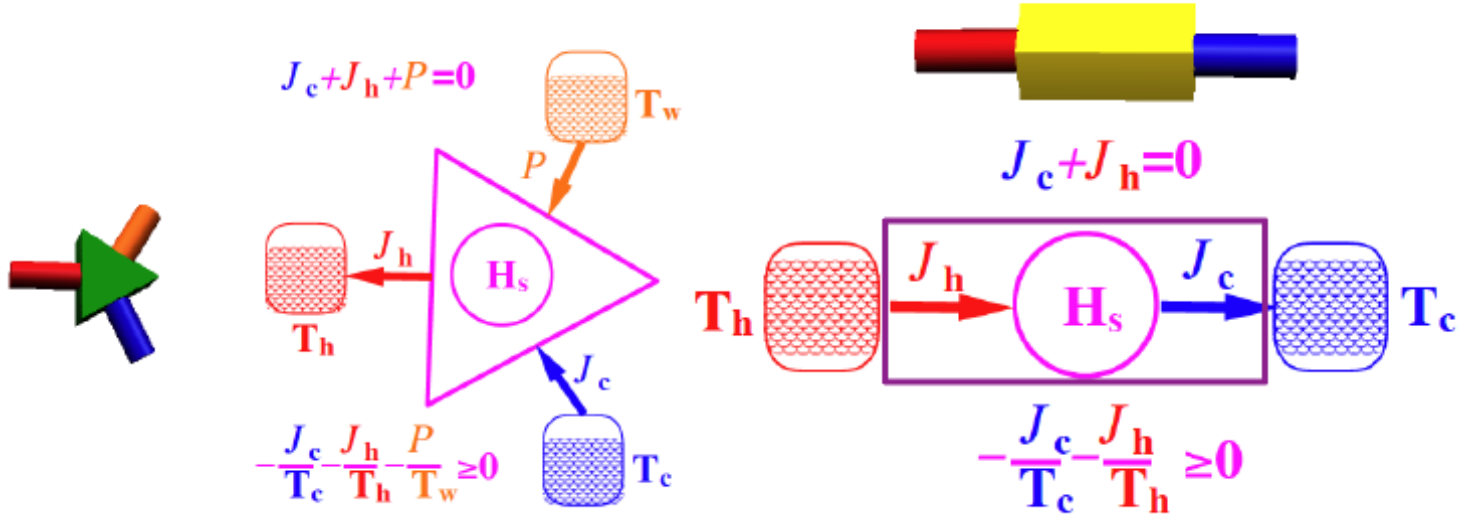

Quantum networks are subject to the Clausius version of the II-law [116]: 
Figure 2. An example of a quantum thermodynamic network composed of wires and tricycles.

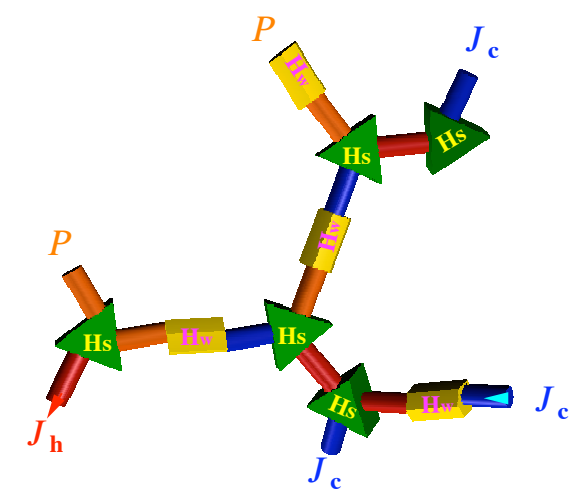

- No process is possible whose sole result is the transfer of heat from a body of lower temperature to a body of higher temperature.

This statement can be generalized to $\mathrm{N}$-coupled heat baths in steady state:

$$
\sum_{j}^{N} \frac{\mathcal{J}_{j}}{T_{j}} \leq 0
$$

A dynamical version of the II-law can be proven, based on Spohn's inequality [117]:

$$
\operatorname{Tr}\{[\mathcal{L} \rho][\ln \rho-\ln \tilde{\rho}]\} \leq 0
$$

which is valid for any LGKS generator, $\mathcal{L}$, with a stationary state, $\tilde{\rho}$.

Computing the time derivative of the von Neumann entropy, $\mathcal{S}_{v n}(t)=-\operatorname{Tr}\{\hat{\boldsymbol{\rho}}(t) \ln \hat{\boldsymbol{\rho}}(t)\}$, and applying Equation (49), one obtains the II-law in the form:

$$
\frac{d}{d t} \mathcal{S}_{v n}(t)-\sum_{j=1}^{M} \frac{\mathcal{J}^{j}(t)}{T_{j}} \geq 0
$$

where: $\mathcal{S}_{v n}(t)=-\operatorname{Tr}\{\hat{\boldsymbol{\rho}}(t) \ln \hat{\boldsymbol{\rho}}(t)\}$.

The heat currents in the steady state for a periodically driven system, $\tilde{\rho}(t)$, are time-independent and given by:

$$
\left.\tilde{\mathcal{J}}^{j}=-T_{j} \sum_{q \in \mathbf{Z}} \sum_{\{\omega \geq 0\}} \operatorname{Tr}\left\{\left(\mathcal{L}_{q \omega}^{j} \tilde{\rho}\right) \ln \tilde{\rho}_{q \omega}^{j}\right)\right\}
$$

In steady-state, they satisfy the II-law in the form:

$$
\sum_{j=1}^{M} \frac{\tilde{\mathcal{J}}^{j}}{T_{j}} \leq 0
$$

while, according to the I-law:

$$
-\sum_{j=1}^{M} \tilde{\mathcal{J}}^{j}=-\tilde{\mathcal{J}}=\overline{\mathcal{P}}
$$


is the averaged power (negative, when the system acts as a heat engine). In the case of a single heat bath, the heat current is always strictly positive, except for the case of no-driving, when it is equal to zero. For the constant Hamiltonian, these formulas are also applicable after removing the index, $q$, which implies

also that $\sum_{j=1}^{M} \tilde{\mathcal{J}}^{j}=0$. In a linear quantum system composed of linearly coupled harmonic oscillators, this statement can be derived directly [115].

External power carries with it zero entropy generation. This is also the case of entropy generation originating from a bath with infinite temperature, $\dot{\mathcal{S}}=-\mathcal{J} /(T \rightarrow \infty)=0$. Furthermore, power obtained from pure Gaussian white noise carries with it zero entropy generation. Another source of zero entropy is a weak quantum measurement [118,119]. It seems counterintuitive, but a refrigerator driven by external power, a high temperature bath, by white noise or by quantum measurements is all equivalent from a thermodynamic standpoint [71]. An illuminating example is of work converted to heat in a driven two-level-system coupled to a bath. Work is a zero entropy source; therefore, it will generate a current flowing to the bath at any finite temperature. Consistency with the second law demand that the work will be dissipated to heat in the bath. In 1946, Bloch proposed to describe the motion of the components of the macroscopic nuclear polarization, $\mathcal{M}$, subject to an external, time-dependent magnetic field [120]. The Bloch equations have been extended to the optical regime, becoming the workhorse of spectroscopy [121]. The Bloch equations have been derived and re-derived based on the weak system-bath coupling limit [122]. Their form has the LGKS structure. Surprisingly, these sets of equations for strong driving fields violate the II-law [102]. The reason for this violation is that in these derivations, the energy levels of the system are not dressed by the external driving field. A derivation of a generalized master equation within the Floquet theory restores the II-law [102,123].

A similar problem arises for the tricycle case. The three-level laser is such an example. The equivalence of the three-level laser with the Carnot engine was first derived by Scovil et al. [2,3]. A dynamical set of equations for the laser, known as Lamb's equation [124], also violates the II-law [51]. Again, the remedy is a dressed state framework for deriving the dissipative LGKS generator $[51,80]$. An interesting case is a tricycle driven by Poissonian noise. Such noise has a unitary component, which effectively modifies the Hamiltonian of the system. As a result, the detailed balance conditions of the hot and cold baths are modified [70]. Only this procedure saves the II-law of thermodynamics.

The warning emerging from these examples is that a careful derivation of the LGKS generator is required in order to be consistent with the II-law of thermodynamics. Examples of violations have been published [125], which could be traced to a flaw in the master equation.

\subsection{Approach to Steady State: Limit Cycle}

In a quantum network, the quantum thermodynamic framework leads to a steady state solution: the limit cycle. The monotonic approach to steady state can be proven for an evolution generated by a completely positive map, $\Lambda$.

$$
\hat{\boldsymbol{\rho}}_{f}=\Lambda \hat{\boldsymbol{\rho}}_{i}
$$

For such a map, the conditional entropy:

$$
\mathcal{S}\left(\hat{\boldsymbol{\rho}} \mid \hat{\boldsymbol{\rho}}_{f}\right)=\operatorname{Tr}\left\{\hat{\boldsymbol{\rho}}\left(\log \hat{\boldsymbol{\rho}}-\log \hat{\boldsymbol{\rho}}_{f}\right)\right\}
$$


is always decreasing [126]:

$$
\mathcal{S}\left(\Lambda \hat{\boldsymbol{\rho}} \mid \Lambda \hat{\boldsymbol{\rho}}_{f}\right) \leq \mathcal{S}\left(\hat{\boldsymbol{\rho}} \mid \hat{\boldsymbol{\rho}}_{f}\right)
$$

If $\hat{\boldsymbol{\rho}}_{f}$ is an invariant of the map, $\Lambda$, then repeated application of the map will lead to a limit, $\hat{\boldsymbol{\rho}} \rightarrow \hat{\boldsymbol{\rho}}_{f}[127,128]$. A quantum network whose generator is a sum of LGKS generators will reach steady state. A reciprocating quantum engine can be described by a completely positive map, $\mathcal{U}$, which is the product of the maps of each segment; for example, a map of the Otto refrigerators, $\mathcal{U}=\mathcal{U}_{h} \mathcal{U}_{h c} \mathcal{U}_{c} \mathcal{U}_{c h}[26,129]$. The above argument means that such cycles will reach a limit cycle of operation [26].

\subsection{The Quantum and Thermodynamic Adiabatic Conditions and Quantum Friction}

Thermodynamic adiabatic processes have no entropy change. Typically, an external control modifies the state. A quantum version of an adiabatic process can be modeled by an externally controlled timedependent Hamiltonian $\hat{\mathbf{H}}(t)$. If the system is isolated, the dynamics are unitary, and therefore, $\mathcal{S}_{v n}$ is a constant. For this reason, the relevant entropy measure for quantum adiabatic processes is the energy entropy, $\mathcal{S}_{E}$, cf. Equation (46). A quantum adiabatic process is defined by the $\mathcal{S}_{E}$ constant. Taking the time derivative leads to:

$$
\frac{d \mathcal{S}_{E}}{d t}=-\sum_{j} \dot{p}_{j} \log p_{j}
$$

where $p_{j}$ is the expectation of the projection on the instantaneous energy level, $\epsilon_{j}(t)$. The adiabatic condition is therefore equivalent to no net change in the population of the instantaneous energy levels. This implies that the Hamiltonian should commute with itself at different times: $\left[\hat{\mathbf{H}}(t), \hat{\mathbf{H}}\left(t^{\prime}\right)\right]=0$.

A generic control Hamiltonian used to modify the system from an initial to a final state is typically constructed from a static "drift" Hamiltonian, $\hat{\mathbf{H}}_{0}$, and a time-dependent control part, $\hat{\mathbf{H}}_{C}(t)$ :

$$
\hat{\mathbf{H}}(t)=\hat{\mathbf{H}}_{0}+\hat{\mathbf{H}}_{C}(t)
$$

Typically, $\left[\hat{\mathbf{H}}_{0}, \hat{\mathbf{H}}_{C}(t)\right] \neq 0$; therefore, also, $\left[\hat{\mathbf{H}}(t), \hat{\mathbf{H}}\left(t^{\prime}\right)\right] \neq 0$, and time ordering corrections mean that strictly adiabatic processes are impossible. Approximate adiabatic dynamics require, therefore, a slow change of the control Hamiltonian, $\hat{\mathbf{H}}_{C}(t)$. These conditions are defined by the adiabatic parameter, $\mu=\sum_{i j}\left|\dot{\omega}_{i j} / \omega_{i j}^{2}\right| \ll 1$, where $\omega_{i j}=\hbar\left(\epsilon_{i}-\epsilon_{j}\right)$ are the instantaneous Bohr frequencies [130].

The adiabatic condition is an important idealization in thermodynamics. For example, if the initial state is the ground state, then the adiabatic conditions define the path that will require minimum work to reach the final value of the control, which will be the ground state of the modified Hamiltonian. This statement can be generalized for an initial thermal state of a harmonic oscillator and a two-level system (TLS), which will commute with $\hat{\mathbf{H}}(t)$ and maintain a thermal state at all times [32].

When the adiabatic conditions are not fulfilled, additional work is required to reach the final control value. For an isolated system, this work is recoverable, since the dynamics is unitary and can be reversed. The coherences stored in the off-diagonal elements of the density operator carry the required information to recover the extra energy cost and reverse the dynamics. Typically, this energy is not recoverable, due to interaction with a bath that causes energy dephasing. The bath, in this case, acts like a measuring apparatus of energy. This lost energy is the quantum version of friction [27,36,37]. The deviation form 
adiabatic behavior can be related to the difference between the energy entropy and the von Neumann entropy [129].

There are several strategies to minimize the effect of quantum friction. One possibility, termed quantum lubrication, is to force the state of the system to commute with the instantaneous Hamiltonian, $[\hat{\boldsymbol{\rho}}(t), \hat{\mathbf{H}}(t)]=0$. This can be achieved by adding an external source of phase noise [38]. It was found that lubrication could be achieved in a small window of control parameters. Outside this window, the noise caused additional heating of the system. In the case of quantum refrigerators, this noise was always harmful, leading to a minimum temperature that the refrigerator can reach $[33,131]$.

Is it possible to find non-adiabatic control solutions with an initial and final state diagonal in the energy representation, $\left[\hat{\boldsymbol{\rho}}_{i}, \hat{\mathbf{H}}(0)\right]=0,\left[\hat{\boldsymbol{\rho}}_{f}, \hat{\mathbf{H}}\left(t_{f}\right)\right]=0$ ? This possibility, which relies on special dynamical symmetries, has been termed shortcut to adiabaticity [32,40-44,132]. The idea is to optimize the scheduling function, $f(t)$, of the control, $\hat{\mathbf{H}}_{C}(t)=\hat{\mathbf{V}}_{C} f(t)$, in such a way that in the shortest time, the frictionless transformation from an initial value of the control function to a final value is achieved.

\section{The III-Law}

Two independent formulations of the III-law of thermodynamics exist, both originally stated by Nernst [133-135]. The first is a purely static (equilibrium) one, also known as the "Nernst heat theorem", phrased:

- The entropy of any pure substance in thermodynamic equilibrium approaches zero as the temperature approaches zero.

The second formulation is dynamical, known as the unattainability principle:

- It is impossible by any procedure, no matter how idealized, to reduce any assembly to absolute zero temperature in a finite number of operations [135,136].

There is an ongoing debate on the relations between the two formulations and their relation to the II-law regarding which, if at all, one of these formulations implies the other [137-140]. Quantum considerations can illuminate these issues.

At steady state, the second law implies that the total entropy production is non-negative, cf. Equation (52). When the cold bath approaches absolute zero temperature, it is necessary to eliminate the entropy production divergence at the cold side. The entropy production at the cold bath when $T_{c} \rightarrow 0$ scales as:

$$
\dot{S}_{c} \sim-T_{c}^{\alpha}, \quad \alpha \geq 0
$$

For the case when $\alpha=0$, the fulfillment of the second law depends on the entropy production of the other baths, which should compensate for the negative entropy production of the cold bath. The first formulation of the III-law slightly modifies this restriction. Instead of $\alpha \geq 0$, the III-law imposes $\alpha>0$, guaranteeing that at absolute zero temperature, the entropy production at the cold bath is zero: $\dot{S}_{c}=0$. This requirement leads to the scaling condition of the heat current, $\mathcal{J}_{c} \sim T_{c}^{\alpha+1}$.

The second formulation is a dynamical one, known as the unattainability principle; no refrigerator can cool a system to absolute zero temperature at finite time. This formulation is more restrictive, imposing 
limitations on the system bath interaction and the cold bath properties when $T_{c} \rightarrow 0$ [71]. The rate of temperature decrease of the cooling process should vanish, according to the characteristic exponent, $\zeta$ :

$$
\frac{d T_{c}(t)}{d t} \sim-T_{c}^{\zeta}, \quad T_{c} \rightarrow 0
$$

Solving Equation (60) leads to:

$$
T_{c}(t)^{1-\zeta}=T_{c}(0)^{1-\zeta}-c t, \zeta<1
$$

where $c$ is a positive constant. From Equation (61), the cold bath is cooled to zero temperature at finite time for $\zeta<1$. The III-law requires, therefore, $\zeta \geq 1$. In order to evaluate Equation (60), the heat current can be related to the temperature change:

$$
\mathcal{J}_{c}\left(T_{c}(t)\right)=-c_{V}\left(T_{c}(t)\right) \frac{d T_{c}(t)}{d t}
$$

This formulation takes into account the heat capacity, $c_{V}\left(T_{c}\right)$, of the cold bath. $c_{V}\left(T_{c}\right)$ is determined by the behavior of the degrees of freedom of the cold bath at low temperature. Therefore, the scaling exponents can be related, $\zeta=1+\alpha-\eta$, where $c_{V} \sim T_{c}^{\eta}$, when $T_{c} \rightarrow 0$.

To get additional insight, specific cases are examined. The quantum refrigerator models differ in their operational mode, being either continuous or reciprocating. When $T_{c} \rightarrow 0$, the refrigerators have to be optimized, adjusting to the decreasing temperature. The receiving mode of the refrigerator has to become occupied to transfer energy. The rate of this process is proportional to a Boltzmann term, $\omega_{c}^{\gamma} \exp \left[-\frac{\hbar \omega_{c}}{k_{B} T_{c}}\right]$. When optimized for maximum cooling rate, the energy difference of the receiving mode should scale linearly with temperature, $\omega_{c} \sim T_{c}[32,52,53,70,71]$. Once optimized, the cooling power of all refrigerators studied have the same dependence on the coupling to the cold bath. This means that the III-law depends on the scaling properties of the heat conductivity, $\gamma_{c}\left(T_{c}\right)$, and the heat capacity, $c_{V}\left(T_{c}\right)$, as $T_{c} \rightarrow 0$.

\subsection{Harmonic Oscillator Cold Heat Bath}

The harmonic heat bath is a generic type of quantum bath. It includes the electromagnetic field: (a photon bath), or a macroscopic piece of solid; (a phonon bath), or Bogoliubov excitations in a BoseEinstein condensate. The model assumes linear coupling of the refrigerator to the bath. The standard form of the bath's Hamiltonian is:

$$
H_{\text {int }}=\left(b+b^{\dagger}\right)\left(\sum_{k}\left(g(k) a(k)+\bar{g}(k) a^{\dagger}(k)\right)\right), H_{B}=\sum_{k} \omega(k) a^{\dagger}(k) a(k)
$$

where $a(k), a^{\dagger}(k)$ are annihilation and creation operators for a mode, $k$. For this model, the weak coupling limit procedure leads to the LGKS generator with the cold bath relaxation rate given by:

$$
\gamma_{c} \equiv \gamma_{c}\left(\omega_{c}\right)=\pi \sum_{k}|g(k)|^{2} \delta\left(\omega(k)-\omega_{c}\right)\left[1-e^{-\frac{\hbar \omega(k)}{k_{B} T_{c}}}\right]^{-1}
$$

For the bosonic field in $d$-dimensional space, with the linear low-frequency dispersion law $(\omega(k) \sim|k|)$, the following scaling properties for the cooling rate at low frequencies are obtained:

$$
\gamma_{c} \sim \omega_{c}^{\kappa} \omega_{c}^{d-1}\left[1-e^{-\hbar \omega_{c} / k_{B} T_{c}}\right]^{-1}
$$


where $\omega_{c}^{\kappa}$ represents the scaling of the coupling strength, $|g(\omega)|^{2}$, and $\omega_{c}^{d-1}$ the scaling of the density of modes. It implies the following scaling relation for the cold current:

$$
\mathcal{J}_{c} \sim T_{c}^{d+\kappa}\left[\frac{\omega_{c}}{T_{c}}\right]^{d+\kappa} \frac{1}{e^{\hbar \omega_{c} / k_{B} T_{c}}-1}
$$

Optimization of Equation (66) with respect to $\omega_{c}$ leads to the frequency tuning, $\omega_{c} \sim T_{c}$, and the final current scaling:

$$
\mathcal{J}_{c}^{o p t} \sim T_{c}^{d+\kappa}
$$

At low temperatures, the heat capacity of the bosonic systems scales like:

$$
c_{V}\left(T_{c}\right) \sim T_{c}^{d}
$$

which produces the scaling of the dynamical equation, [ Equation (60) ]:

$$
\frac{d T_{c}(t)}{d t} \sim-\left(T_{c}\right)^{\kappa}
$$

Similarly, the same scaling [ Equation (69)] achieved for the periodically driven refrigerator, with the optimization tuning, $\omega_{c}, \lambda \propto T_{c}$.

The III-law implies a constraint on the form of interaction with a bosonic bath:

$$
\kappa \geq 1
$$

For standard systems, like electromagnetic fields or acoustic phonons with the linear dispersion law, $\omega(k)=v|k|$, and the formfactor, $g(k) \sim|k| / \sqrt{\omega(k)}$, the parameter, $\kappa=1$; as for low $\omega,|g(\omega)|^{2} \sim|k|$. However, the condition in Equation (70) excludes exotic dispersion laws, $\omega(k) \sim|k|^{\alpha}$ with $\alpha<1$, which produce infinite group velocity forbidden by relativity theory anyway. Moreover, the popular choice of Ohmic coupling is excluded for systems in dimension $d>1$. The condition in Equation (70) can also be compared with the condition:

$$
\kappa>2-d
$$

which is necessary to assure the existence of the ground state for the bosonic field interacting by means of the Hamiltonian in Equation (63). The third law loses its validity if the cold bath does not have a ground state. For a harmonic bath, this could happen if even one of the effective oscillators has an inverted potential.

\subsection{The Existence of a Ground State}

A natural physical stability condition, which should be satisfied by any model of an open quantum system, is that its total Hamiltonian should be bounded from below and should possess a ground state. In the quantum degenerate regime, even a mixture of isotopes will segregate and lead to a unique ground state. In the case of systems coupled linearly to bosonic heat baths, it implies the existence of the ground state for the following bosonic Hamiltonian (compare with [63]):

$$
H_{b o s}=\sum_{k}\left\{\omega(k) a^{\dagger}(k) a(k)+\left(g(k) a(k)+\bar{g}(k) a^{\dagger}(k)\right)\right\}
$$


Introducing a formal transformation to a new set of bosonic operators:

$$
a(k) \mapsto b(k)=a(k)+\frac{\bar{g}(k)}{\omega(k)}
$$

we can write:

$$
H_{\text {bos }}=\sum_{k} \omega(k) b^{\dagger}(k) b(k)-E_{0}, E_{0}=\sum_{k} \frac{|g(k)|^{2}}{\omega(k)}
$$

with the formal ground state $|0\rangle$ satisfying:

$$
b(k)|0\rangle=0, \text { for all } k
$$

For the interesting case of an infinite set of modes, $\{k\}$, labeled by the $d$-dimensional wave vectors, two problems can appear:

(1) The ground state energy, $E_{0}$, can be infinite, i.e., it does not satisfy:

$$
\sum_{k} \frac{|g(k)|^{2}}{\omega(k)}<\infty
$$

(2) The transformation in Equation (73) can be implemented by a unitary one, i.e., $b(k)=U a(k) U^{\dagger}$, if and only if:

$$
\sum_{k} \frac{|g(k)|^{2}}{\omega(k)^{2}}<\infty
$$

Non-existence of such a unitary implies non-existence of the ground state (75) (in the Fock space of the bosonic field) and is called the van Hove phenomenon [141].

While the divergence of the sums Equations (76) and (77) (or integrals for the infinite volume case) for large $|k|$ can be avoided by placing an ultra-violet cutoff, the stronger condition in Equation (77) imposes restrictions on the form of $g(k)$ at low frequencies. Assuming that $\omega(k)=v|k|$ and $g(k) \equiv g(\omega)$, the condition in Equation (77) is satisfied for the following low-frequency scaling in the $d$-dimensional case:

$$
|g(\omega)|^{2} \sim \omega^{\kappa}, \kappa>2-d
$$

These conditions on the dispersion relation of the cold bath required for a ground state are identical to the conditions for the III-law Equation (71). Consistency with the III-law ensures the existence of the ground state.

\subsection{Ideal Bose/Fermi Gas Cold Heat Bath}

An important generic cold bath consists of a degenerate quantum gas composed of ideal Bose or Fermi gas. The model refrigerator consists of the working medium of (infinitely) heavy particles with the internal structure approximated (at least at low temperatures) by a two-level system (TLS) immersed in the low density gas at the temperature, $T_{c}$. Insight into the III-law comes from realizing that the degenerate gas is in equilibrium with a normal part. The external refrigerator only couples to the normal part. Once the temperature approaches zero, the fraction of the normal part decreases, eventually nulling the cooling current. Another source of excitations are collective excitations of the Bogolubov type [142]. The low energy tail can be described as a phonon bath with linear dispersion, as in the previous section. 
The Markovian dynamics of such systems were derived by Dumcke [143] in the low density limit and $N$-level internal structure. For the case of the TLS, there is one receiving Bohr frequency, $\omega_{c}$. Cooling occurs due to the inelastic scattering, leading to energy exchange with this frequency [71] so that:

$$
\gamma_{c}=2 \pi \mathbf{n} \int d^{3} \vec{p} \int d^{3} \overrightarrow{p^{\prime}} \delta\left(E\left(\overrightarrow{p^{\prime}}\right)-E(\vec{p})-\hbar \omega_{c}\right) f_{T_{c}}\left(\vec{p}_{g}\right)\left|T\left(\overrightarrow{p^{\prime}}, \vec{p}\right)\right|^{2}
$$

with $\mathbf{n}$, the particle density, $f_{T_{c}}\left(\vec{p}_{g}\right)$, the probability distribution of the gas momentum strictly given by Maxwell's distribution, $\vec{p}$ and $\overrightarrow{p^{\prime}}$, the incoming and outgoing gas particle momenta and $E(\vec{p})=p^{2} / 2 m$ the kinetic energy of the gas particle.

At low-energies (low-temperature), scattering of neutral gas particle at 3-d can be characterized by s-wave scattering length, $a_{s}$, having a constant transition matrix, $|T|^{2}=\left(\frac{4 \pi a_{s}}{m}\right)^{2}$. For this model, the integral in Equation (79) is calculated:

$$
\gamma_{c}=(4 \pi)^{4}\left(2 \pi m T_{c}\right)^{-\frac{1}{2}} a_{s}^{2} \mathbf{n} \omega_{c} \mathcal{K}_{1}\left(\frac{\hbar \omega_{c}}{2 k_{B} T_{c}}\right) e^{\frac{\hbar \omega_{c}}{2 k_{B} T_{c}}}
$$

where $\mathcal{K}_{p}(x)$ is the modified Bessel function of the second kind. Notice that Equation (80) is also valid for a harmonic oscillator instead of TLS, assuming only linear terms in the interaction and using the Born approximation for the scattering matrix.

Optimizing Equation (62) with respect to $\omega_{c}$ leads to $\omega_{c} \sim T_{c}$. Then, the scaling of the heat current becomes:

$$
\mathcal{J}_{c}^{\text {opt }} \sim \mathbf{n}\left(T_{c}\right)^{\frac{3}{2}}
$$

When the Bose gas is above the critical temperature for the Bose-Einstein condensation, the heat capacity, $c_{V}$, and the density, $\mathbf{n}$, are constants. Below the critical temperature, the density, $\mathbf{n}$, in Equation (79) should be replaced with the density, $\mathbf{n}_{e x}$, of the exited states, having both $c_{V}, \mathbf{n}_{e x}$ scale as $\sim\left(T_{c}\right)^{\frac{3}{2}}$, which finally implies that:

$$
\frac{d T_{c}(t)}{d t} \sim-\left(T_{c}\right)^{\frac{3}{2}}
$$

In the case of Fermi gas at low temperatures, only the small fraction, $\mathbf{n} \sim T_{c}$, of fermions participate in the scattering processes and contribute to the heat capacity; the rest is "frozen" in the "Dirac sea", below the Fermi surface. Again, this effect modifies, both sides of Equation (60), in the same way, and therefore, Equation (82) is consistent with the III-law. Similarly, a possible formation of Cooper pairs below the critical temperature does not influence the scaling in Equation (82).

Figure 3 demonstrates the III-law, showing the vanishing of the cooling current, $\mathcal{J}_{c}$, and the temperature decrease rate, $\frac{d T_{c}}{d t}$, as a function of $T_{c}$ for the cases of the harmonic bath and Bose gas bath. 
Figure 3. A demonstration of the III-law shown as the vanishing of the cooling current and the rate of temperature decrease as $T_{c} \rightarrow 0$. The harmonic bath in 3-d is indicated in blue, and the Bose gas in 3-d in red. The Bose gas cools faster when $T_{c} \rightarrow 0$, but its rate of temperature decrease is slower than the harmonic bath.
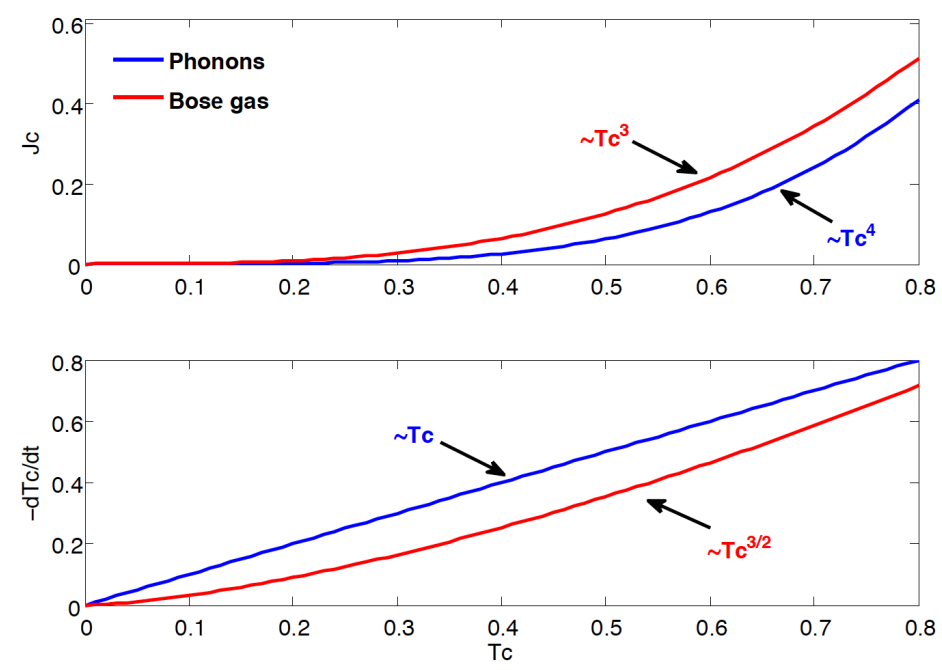

\subsection{Thermoelectric Refrigerators}

The thermoelectric effect is a combined charge and heat flow between two or more reservoirs [144]. The non-linear interaction that allows such a device to operate is supplied by the coupling between these currents. The first law is modified by the electrical power, $\mathcal{P}_{j}^{e}=V_{j} I_{j}$, where $V_{j}$ is the bias voltage and $I_{j}$ is the electrical current. The I-law becomes:

$$
\frac{d E_{S}}{d t}=\sum_{j}^{N} \mathcal{J}_{j}+\mathcal{P}_{j}^{e}
$$

The II-law is not modified: based on scattering theory, the charge redistribution does not change the entropy [145].

The maximum heat current that can be extracted from the cold bath is limited by [144]:

$$
\mathcal{J}_{c} \leq \frac{\pi^{2}}{6 \hbar^{2}} N_{c}\left(k_{b} T_{c}\right)^{2}
$$

where $N_{c}$ is the number of scattering channels. This means that the scaling of the heat current is $\mathcal{J}_{c} \sim T_{c}^{2}$, and for fermions, the heat capacity scales as $c_{V} \sim T_{c}$. Therefore, consistency with the III-law is obtained with the exponent, $\zeta=1$ in Equation (60).

The dynamical version of the III-law is up for critical analysis [71]. The examples of quantum refrigerators show that the cooling exponents are independent of the type of refrigerator model used. The III-law exponents depend on the cold bath characteristics, the ratio between the heat conductivity and the heat capacity for a specific bath. This ratio should scale as $\sim T_{c}^{\zeta}, \zeta>1$ for the III-law to hold, cf. Equation (60). There has been a recent challenge to the III-law, claiming that zero temperature can be reached $[80,146]$. The present view advocates that this discrepancy is caused by an uncontrolled approximation, leading to the particular dispersion used. 


\section{Conclusions}

A dynamical view of quantum thermodynamics was presented. The theory is based on a series of idealizations. The main one is to impose a local structure through thermodynamic partitions. If the dynamics of the universe are generated by a Hamiltonian, then the conservation of energy and conservation of von Neumann entropy are trivial statements. However, without partitions, local information about the world cannot be extracted. Our goal is to learn about our local environment. Quantum thermodynamics follows the laws of thermodynamics within a local structure. The theory of open quantum systems is employed to construct such partitions. The weak coupling limit is an idealization employed to construct isothermal partitions, which are consistent with the first and second law of thermodynamics.

Quantum thermodynamics is applicable at the level of a single particle. This means that very simple models have the same thermodynamic characteristics as macroscopic devices. For example, efficiency at maximum power related to finite-time-thermodynamics. Furthermore, the quantum and thermodynamic adiabatic behavior are closely linked. Deviations lead to friction, resulting in reduced efficiency.

The III-law can be thought of as an attempt to completely isolate a subsystem. Once a system is cooled to absolute zero temperature, it reaches a pure ground state and, therefore, becomes disentangled from the rest of the universe. The III-law is a statement that obtaining an isolated pure state is an idealization impossible at finite time.

This review advocates the view that the laws of thermodynamics are true in any quantum circumstance. An apparent failure of a quantum model is caused by flaws in the approximation, usually in the derivation of the master equations. One can therefore use thermodynamics as a consistency check for approximate quantum theories.

\section{Acknowledgments}

I want to thank Eitan Geva, Tova Feldmann, Jose P. Palao, Yair Rezek, Michael Khasin, Amikam Levy, Peter Salamon, Gershon Kurizki, Lajos Diosi and Robert Alicki for sharing their insight on this subject. Work supported by the Israel Science Foundation. Work carried out at the Fritz Haber research center for molecular dynamics, Hebrew University, Jerusalem, at ITAMP Institute for Theoretical Atomic Molecular and Optical Physics, Harvard-Smithsonian, Cambridge, MA, and at KITP, Kalvy Institute for Theoretical Physics, UCSB, CA. I thank the hospitality of these institutes.

\section{References}

1. Einstein, A. Über einen die Erzeugung und Verwandlung des Lichtes betreffenden heuristischen Gesichtspunkt (On a Heuristic Viewpoint Concerning the Production and Transformation of Light). Annalen der Physik 1905, 17, 132.

2. Scovil, H.E.; du Bois, E.O.S. Three-level masers as heat engines. Phys. Rev. Lett. 1959, 2, 262-263.

3. Geusic, J.; du Bois, E.O.S.; Grasse, R.D.; Scovil, H.E. Quantum equivalence of the carnot cycle. Phys. Rev. 1967, 156, 343-351. 
4. Carnot, S. Réflections sur la Puissance Motrice du Feu et sur les Machines propres à Développer cette Puissance; Bachelier: Paris, France, 1824.

5. Lindblad, G. On the generators of quantum dynamical semigroups. Comm. Math. Phys. 1976, 48, 119-130.

6. Gorini, V.; Kossakowski, A.; Sudarshan, E.C.G. Completely positive dynamical semigroups of N-level systems. J. Math. Phys. 1976, 17, 821-825.

7. Alicki, R. Quantum open systems as a model of a heat engine. J. Phys A: Math. Gen. 1979, 12, L103-L107.

8. Kosloff, R. A quantum mechanical open system as a model of a heat engine. J. Chem. Phys. 1984, 80, 1625-1631.

9. Curzon, F.; Ahlborn, B. Efficiency of a Carnot engine at maximum power output. Am. J. Phys. 1975, 43, 22-24.

10. Andresen, B.; Salamon, P.; Berry, R.S. Thermodynamics in finite time. Phys. Today 1984, $37,62-70$.

11. Salamon, P.; Nulton, J.D.; Siragusa, G.; Andersen, B.; Limon, A. Principles of control thermodynamics. Energy 2001, 26, 307-319.

12. Geva, E.; Kosloff, R. A quantum mechanical heat engine operating in finite time. A model consisting of spin-1/2 systems as the working fluid. J. Chem. Phys. 1992, 96, 3054-3067.

13. Lloyd, S. Quantum-mechanical Maxwells demon. Phys. Rev. A 1997, 56, 3374-3382.

14. Bender, C.M.; Brody, D.C.; Meister, B.K. Entropy and temperature of a quantum Carnot engine. Proc. Roy. Soc. Lond. A 2002, 458, 1519-1526.

15. He, J.; Chen, J.; Hua, B. Quantum refrigeration cycles using spin-1/2 systems as working substance. Phys. Rev. E 2002, 65, 036145.

16. Quan, H.T.; Liu, Y.X.; Sun, C.P.; Nori, F. Quantum thermodynamic cycles and quantum heat engines. Phys. Rev. E 2007, 76, 031105.

17. Guo, J.; Zhang, X.; Su, G.; Chen, J. The performance analysis of a micro-/nanoscaled quantum heat engine. Phys. Stat. Mech. Appl. 2012, 391, 6432-6439.

18. Feldmann, T.; Geva, E.; Kosloff, R.; Salamon, P. Heat engines in finite time governed by master equations. Am. J. Phys. 1996, 64, 485-492.

19. Feldmann, T.; Kosloff, R. Performance of discrete heat engines and heat pumps in finite time. Phys. Rev. E 2000, 61, 4774-4790.

20. Wang, H.; Liu, S.Q.; He, J.Z. Thermal entanglement in two-atom cavity QED and the entangled quantum Otto engine. Phys. Rev. E 2009, 79, 041113.

21. He, J.; Xian, H.; Wei, T. The performance characteristics of an irreversible quantum Otto harmonic refrigeration cycle. Sci. China Ser. G Phys. Mech. Astron. 2009, 52, 1317-1323.

22. Henrich, M. J.; Rempp, F.; Mahler, G. Quantum thermodynamic Otto machines: A spin-system approach. Eur. Phys. J. 2005, 151, 157-165.

23. Jahnke, T. ; Birkov, J.; Mahler, G. On the nature of thermodynamic extremum principles: The case of maximum efficiency and maximum work. Ann. Phys. 2008, 17, 88-100.

24. Allahverdyan, A.E.; Johal, R.S.; Mahler, G. Work extremum principle: Structure and function of quantum heat engines. Phys. Rev. E 2008, 77, 041118. 
25. Gemmer, J.; Mechel, M.; Mahler, G. Quantum Thermodynamics; Springer: Berlin, Germany, 2009.

26. Feldmann, T.; Kosloff, R. Characteristics of the limit cycle of a reciprocating quantum heat engine. Phys. Rev. E 2004, 70, 046110.

27. Rezek, Y.; Kosloff, R. Irreversible performance of a quantum harmonic heat engine. New J. Phys. 2006, 8,83 .

28. Abah, O.; Rossnagel, J.; Jacob, G.; Deffner, S.; Schmidt-Kaler, F.; Singer K.; Lutz, E. Single-ion heat engine at maximum power. Phys. Rev. Lett. 2012, 109, 203006.

29. Thomas, G.; Johal, R. Coupled quantum Otto cycle. Phys. Rev. E 2011, 83, 031135.

30. He, X.; He, J. Thermal entangled four-level quantum Otto heat engine. Sci. China Phys. Mech. Astron. 2012, 55, 1751-1756.

31. Wang, R.; Wang, J.; He, J.; Ma, Y. Efficiency at maximum power of a heat engine working with a two-level atomic system. Phys. Rev. E 2013, 87, 042119.

32. Rezek, R.; Salamon, P.; Hoffmann, K.H.; Kosloff, R. The quantum refrigerator: The quest for the absolute zero. Euro. Phys. Lett. 2009, 85, 30008.

33. Feldmann, T.; Kosloff, R. Minimal temperature of quantum refrigerators. Euro. Phys. Lett. 2010, 89, 20004.

34. Rempp, F.; Michel, M.; Mahler, G. Cyclic cooling algorithm. Phys. Rev. A 2007, 76, 032325.

35. Kaufman, A.M.; Lester B.J.; Regal, C.A. Cooling a single atom in an optical tweezer to its quantum ground state. Phys. Rev. X 2012, 2, 041014.

36. Kosloff, R.; Feldmann, T. A discrete four stroke quantum heat engine exploring the origin of friction. Phys. Rev. E 2002, 65, 055102.

37. Feldmann, T.; Kosloff, R. The quantum four stroke heat engine: Thermodynamic observables in a model with intrinsic friction. Phys. Rev. E 2003, 68, 016101.

38. Feldmann, T.; Kosloff, R. Quantum lubrication: Suppression of friction in a first-principles four-stroke heat engine. Phys. Rev. E 2006, 73, 025107(R).

39. Diósi, L.; Feldmann, T.; Kosloff, R; On exact identity between thermodynamic and informatic entropies in a unitary model of friction. Int. J. Quantum Inf. 2006, 4, 99-104.

40. Chen, X.; Ruschhaupt, A.; Schmidt, S.; del Campo, A.; Guery-Odelin, D.; Muga, J.G. Fast optimal frictionless atom cooling in harmonic traps. Phys. Rev. Lett. 2010, 104, 063002.

41. Chen, X.; Lizuain, I.; Ruschhaupt, A.; Gury-Odelin, D.; Muga, J.G. Shortcut to adiabatic passage in two- and three-level atoms. Phys. Rev. Lett. 2010, 105, 123003.

42. Ibez, S.; Chen, X.; Torrontegui, E.; Muga, J.G.; Ruschhaupt, A. Multiple schrdinger pictures and dynamics in shortcuts to adiabaticity. Phys. Rev. Lett. 2012, 109, 100403.

43. Salamon, P.; Hoffmann, K.H.; Rezek, Y.; Kosloff, R. Maximum work in minimum time from a conservative quantum system. Phys. Chem. Chem. Phys. 2009, 11, 1027-1032.

44. Hoffmann, K.H.; Salamon, P.; Rezek, Y.; Kosloff, R. Time-optimal controls for frictionless cooling in harmonic traps. Euro. Phys. Lett. 2011, 96, 60015.

45. Salamon, P.; Nitzan, A.; Andresen, B.; Berry, R.S. Minimum entropy production and the optimization of heat engines. Phys. Rev. A 1980, 21, 2115-2129. 
46. Bejan, A. Entropy Generation Minimization ; Chemical Rubber Corp: Cleaveland, OH, USA, 1996.

47. Esposito, M.; Kawai, R.; Lindenberg, K.; van den Brook, C. Efciency at maximum power of low-dissipation carnot engines. Phys. Rev. Lett. 2010, 105, 150603.

48. Van den Brook, C. Efficiency at maximum power in the low-dissipation limit. Eur. Phys. Lett. 2013, 101, 10006.

49. Wang, H. Quantum-mechanical Brayton engine working with a particle in a one-dimensional harmonic trap. Phys. Scripta 2013, 87, 055009.

50. Geva, E.; Kosloff, R. On the classical limit of quantum thermodynamics in finite time. J. Chem. Phys. 1992, 97, 4398-4412.

51. Geva, E.; Kosloff, R. The quantum heat engine and heat pump: An irreversible thermodynamic analysis of the three-level amplifier. J. Chem. Phys. 1996, 104, 7681-7698.

52. Kosloff, R.; Geva, E.; Gordon, J.M. The quantum refrigerator in quest of the absolute zero. $J$. Appl. Phys. 2000, 87, 8093-8097.

53. Palao, P.J.; Kosloff, R.; Gordon, J.M. Quantum thermodynamic cooling cycle. Phys. Rev. E 2001, 64, 056130.

54. Scully, M.O.; Zubairy, M.S.; Agarwal, G.S.; Walther, H. Extracting work from a single heat bath via vanishing quantum coherence. Science 2003, 299, 862-864.

55. Kieu, T.D. The second law, Maxwell's demon, and work derivable from quantum heat engines. Phys. Rev. Lett. 2004, 93, 140403.

56. Segal, D.; Nitzan, A. Molecular heat pump. Phys. Rev. E 2006, 73, 026109.

57. Bushev, P.; Rotter, D.; Wilson, A.; Dubin, F.; Becher, C.; Eschner, J.; Blatt, R.; Steixner, V.; Rabl, P.; Zoller, P. Feedback cooling of a single trapped ion. Phys. Rev. Lett. 2006, 96, 60010.

58. Boukobza, E.; Tannor, D.J. Thermodynamic analysis of quantum light purification. Phys. Rev. A 2008, 78, 013825.

59. Birjukov, J.; Jahnke, T.; Mahler G. Quantum thermodynamic processes: a control theory for machine cycles. Eur. Phys. J. B 2008, 64, 105-108.

60. Segal, D. Vibrational relaxation in the Kubo oscillator: Stochastic pumping of heat. J. Chem. Phys. 2009, 130, 134510.

61. Esposito, M.; Lindberg, K.; van den Brook, C. Thermoelectric efficiency at maximum power in a quantum dot. Eur. Phys. Lett. 2009, 85, 043003.

62. Linden, N.; Popescu, S.; Skrzypczyk, P. How small can thermal machines be? Towards the smallest possible refrigerator. Phys. Rev. Lett. 2010, 105, 130401.

63. Scully, M.O. Quantum photocell: Using quantum coherence to reduce radiative recombination and increase efficiency. Phys. Rev. Lett. 2010, 104, 207701.

64. Svidzinsky, A.A.; Dorfman K.E.; Scully, M.O. Enhancing photovoltaic power by Fano-induced coherence. Phys. Rev. A 2011, 84, 053818.

65. Sothmann, B.; Büttiker, M. Magnon-driven quantum-dot heat engine. Eur. Phys. Lett. 2012, 99, 27001.

66. Geusic, J.E.O.; Schulz-du Bois, R.D.G.; Scovil, H.E. Three Level Spin Refrigeration and Maser Action at 1500mc/sec J. App. Phys. 1959, 30, 1113-1114. 
67. Tsujikawa, I.; Murao, T. Possibility of optical cooling of ruby. J. Phys. Soc. Jpn. 1963, $18,503-510$.

68. Hnsch, T.W.; Schawlow, A.L. Cooling of gases by laser radiation. Opt. Commun. 1975, 13, 6869.

69. Wineland, D.J.; Dehmelt, H. Proposed 1024 upsilon less that upsilon laser fluorescence spectroscopy on T1-Mono-Ion oscillator III. Bull. Am. Phys. Soc. 1975, 20, 637.

70. Levy, A.; Kosloff, R. Quantum absorption refrigerator. Phys. Rev. Lett. 2012, 108, 070604.

71. Levy, A.; Alicki, R.; Kosloff, R. Quantum refrigerators and the third law of thermodynamics. Phys. Rev. E 2012, 85, 061126.

72. Sandner, K.; Ritsch, H. Temperature gradient driven lasing and stimulated cooling. Phys. Rev. Lett. 2012, 109, 93601.

73. Gieseler, J.; Deutsch, B.; Quidant, R.; Novotny, L. Subkelvin parametric feedback cooling of a laser-trapped nanoparticle. Phys. Rev. Lett. 2012, 109, 103603.

74. Guo, J.; Su, G.; Chen, J. The performance evaluation of a micro/nano-scaled cooler working with an ideal Bose gas. Phys. Lett. A 2012, 376, 270-274.

75. Yi, Z.; Gu, W.-J.; Li, G.-X. Sideband cooling of atoms with the help of an auxiliary transition. Phys. Rev. Lett. 2012, 109, 055401.

76. Teufel, J.D.; Donner, T.; Li, D.; Harlow, J.W.; Allman, M.S.; Cicak, K.; Sirois, A.J.; Whittaker, J.D.; Lehnert, K.W.; Simmonds, R.W. Sideband cooling of micromechanical motion to the quantum ground state. Nature 2011, 475, 359-363.

77. Verhagen, E.; Deléglise, S.; Weis, S.; Schliesser A.T.J.; Kippenberg, T.J. Quantum-coherent coupling of a mechanical oscillator to an optical cavity mode. Nature 2012, 482, 63-67.

78. Rahmani, A.; Kitagawa, T.; Demler, E.; Chamon, C. Cooling through optimal control of quantum evolution. Phys. Rev. A 2013, 87, 043607.

79. Pekola, J.P.; Hekking, F.W.J. Normal-Metal-Superconductor tunnel junction as a brownian refrigerator. Phys. Rev. Lett. 2007, 98, 210604.

80. Levy, A.; Alicki, R.; Kosloff, R. Comment on Cooling by Heating: Refrigeration Powered by Photons. Phys. Rev. Lett. 2012, 109, 248901.

81. Cleuren, B.R.; van den Broeck, C. Cooling by heating: Refrigeration powered by photons. Phys. Rev. Lett. 2012, 108, 120603.

82. Gelbwaser-Klimovsky, D.; Alicki, R.; Kurizki, G. Minimal universal quantum heat machine. Phys. Rev. E 2013, 87, 012140.

83. Feingold, M.; Peres, A. Distribution of matrix elements of chaotic systems. Phys. Rev. A 1986, 34, 591-595.

84. Deutsch, J.M. Quantum statistical mechanics in a closed system. Phys. Rev. A 1991, 43, 20462049.

85. Srednicki, M. Chaos and quantum thermalization. Phys. Rev. E 1994, 50, 888-901.

86. Rigol, M.; Dunjko, V.; Olshanii, M. Thermalization and its mechanism for generic isolated quantum systems. Nature 2008, 452, 854-858.

87. Khasin, M.; Kosloff, R. Efficient simulation of quantum evolution using dynamical coarse graining. Phys. Rev. A 2008, 78, 012321. 
88. Khasin, M.; Kosloff, R. Algorithm for simulation of quantum many-body dynamics using dynamical coarse-graining. Phys. Rev. A 2010, 81, 043635.

89. Partovi, M. Quantum Thermodynamics. Physics Letters A 1989, 137, 440-444.

90. Calderbank, A.R.; Shor, P.W. Good quantum error-correcting codes exist. Phys. Rev. A 1996, 54, 1098-1105.

91. Von Neumann, J. Mathematical Foundations of Quantum Mechanics ; Princeton University Press: Princeton, NJ, USA, 1955.

92. Breuer, H.P.; Petruccione, F. Open Quantum Systems; Oxford university press: Oxford, UK, 2002.

93. Davis, E.B. Markovian master equations. Comm. Math. Phys. 1974, 39, 91-110.

94. Davis, E.B. Model of atomic radiation. Ann. Inst. H. Poincaré Phys. Théor. 1978, 28, 91-110.

95. Alicki, R.; Lendi, K. Quantum Dynamical Semigroups and Applications ; Springer-Verlag: Berlin, Germany, 1987.

96. Kraus, K. General State Change in Quantum Theory Ann. Phys. 1971, 64, 311-335.

97. Lindblad, G. On the existence of quantum subdynamics. J. Phys A: Math. Gen. 1996, 29, 4197-4207.

98. Kubo, R.B. Statistical-mechanical theory of irreversible processes. I. General theory and simple applications to magnetic and conduction problems. J. Phys. Soc. Japan 1957, 12, 570-586.

99. Martin, P.C.; Schwinger, J. Theory of many-particle systems. I. Phys. Rev. 1959, 115, 1342-1373.

100. Spohn, H.; Lebowitz, J. Irreversible thermodynamics for quantum systems weakly coupled to thermal reservoirs. Adv. Chem. Phys. 1979, 38, 109.

101. Kosloff, R.; Ratner, M.A. Beyond linear response: Lineshapes for coupled spins or oscillators via direct calculation of dissipated power. J. Chem. Phys. 1984, 80, 2352-2362.

102. Geva, E.; Kosloff, R.; Skinner, J. On the relaxation of a two-level system driven by a strong electromagnetic field. J. Chem. Phys. 1995, 102, 8541-8561.

103. Lieb, E.H.; Yngvason, J. The physics and mathematics of the second law of thermodynamics. Phys. Rep. 1999, 310, 1-96.

104. Kosloff, R. Thermodynamic aspects of the quantum measurement process. Adv. Chem. Phys. 1980, 46, 153-193.

105. Szilard, L. On the minimization of entropy in a thermodynamic system with interferences of intelligent beings. Z. Phys. 1929, 53, 840-856.

106. Brilluin, L. Science and Information Theory; Academic Press: New York, NY, USA, 1956.

107. Maruyama, K.; Nori, F.; Verdal, V. Colloquium: The physics of Maxwell's demon and information. Rev. Mod. Phys. 2009, 81, 1-23.

108. Travis Bannerman, S.; Price, G.N.; Viering, K.; Raizen, M.G. Single-photon cooling at the limit of trap dynamics: Maxwell's demon near maximum efficiency. New J. Phys. 2009, 11, 063044.

109. Nielsen, M.A.; Chuang, I.L. Quantum Computation and Quantum Information; Cambridge University Press: Cambridge, UK, 2000.

110. Polkovnikov, A. Microscopic diagonal entropy and its connection to basic thermodynamic relations. Ann. Phys. 2011, 326, 486-499.

111. Boukobza, E.; Tannor, D.J. Entropy exchange and entanglement in the Jaynes-Cummings model. Phys. Rev. A 2005, 78, 063821. 
112. Horodecki, R.; Horodecki, P.; Horodecki, M.; Horodecki, K. Quantum entanglement. Rev. Mod. Phys. 2007, 81, 865-942.

113. Zurek, W.H. Quantum discord and Maxwells demons. Phys. Rev. A 2003, 67, 012320.

114. Khasin, M.; Kosloff, R. Rise and fall of quantum and classical correlations in open-system dynamics. Phys. Rev. A 2007, 76, 012304.

115. Martinez, E.A.; Paz, J.P. Dynamics and thermodynamics of linear quantum open systems. Phys. Rev. Lett. 2013, 110, 130406.

116. Clausius, R. Ueber Die Bewegende Kraft Der Wrme Und Die Gesetze, Welche Sich Daraus Fr Die Wrmelehre Selbst Ableiten Lassen. Annalen der Physik 1850, 79, 368.

117. Spohn, H.; Lebowitz, J. Irreversible thermodynamics for quantum systems weakly coupled to thermal reservoirs. Adv. Chem. Phys. 1978, 109, 38.

118. Diosi, L. Continuous quantum measurement and ito formalism. Phys. Lett. A 1988, 129, 419423.

119. Diosi, L. A Short Course in Quantum Information Theory-An Approach From Theoretical Physics, 2nd ed.; Lecture Notes in Physics; Springer: Berlin, Germany, 2011; Volume 827, 31-37.

120. Bloch, F.; Hansen W.W.; Packard M. Nuclear induction. Phys. Rev. 1946, 70, 460-474.

121. Allen, L.C.; Eberly, J.H. Optical Resonance and Two-Level Atoms; Wiley, Courier Dover Publications: New York, NY, USA, 1975.

122. Wangsness, R.K.; Bloch, F. The dynamical theory of nuclear induction. Phys. Rev. 1953, 89, 729-739.

123. Szczygielski, K.; Gelbwaser-Klimovsky, D.; Alicki, R. Markovian master equation and thermodynamics of a two-level system in a strong laser field. Phys. Rev. E 2013, 87, 012120.

124. Lamb, W. Theory of an optical maser. Phys. Rev. 1964, 134, A1429-A1450.

125. Allahverdyan, A.E.; Nieuwenhuizen, T.M. Extraction of work from a single thermal bath in the quantum regime. Phys. Rev. Lett. 2000, 85, 1799-1802.

126. Lindblad, G. Expectation and entropy inequalities for finite quantum systems. Comm. Math. Phys. 1974, 39, 111-119.

127. Frigerio, A. Quantum dynamical semigroups and approach to equilibrium. Lett. Math. Phys. 1977, 2, 79-87.

128. Frigerio, A. Stationary states of quantum dynamical semigroups. Comm. Math. Phys. 1978, 63, 269-276.

129. Feldmann, T.; Kosloff, R. Short time cycles of purely quantum refrigerators. Phys. Rev. E 2012, $85,051114$.

130. Comparat, D. General conditions for quantum adiabatic evolution. Phys. Rev. A 2009, $80,012106$.

131. Kosloff, R.; Feldmann, T. Optimal performance of reciprocating demagnetization quantum refrigerators. Phys. Rev. E 2010, 82, 011134.

132. Boldt, F.; Hoffmann, K.H.; Salamon, P.; Kosloff, R. Time-optimal processes for interacting spin systems. Euro. Phys. Lett. 2012, 99, 40002.

133. Nernst, W. Ueber die Berechnung chemischer Gleichgewichte aus thermischen Messungen. Nachr. Kgl. Ges. Wiss. Gött. 1906, 1, 1-40. 
134. Nernst, W. Ub̈er die Beziehung zwischen War̈meentwicklung und maximaler Arbeit bei kondensierten Systemen. Ber. Kgl. Pr. Akad. Wiss. 1906, 52, 933-940.

135. Nernst, W. The Theoretical and Experimental Bases of the New Heat Theorem. [in German, Die Theoretischen und Experimentellen Grundlagen des Neuen War̈mesatzes.]; W. Knapp: Halle, 1918.

136. Fowler, R.H.; Guggenheim, E.A. Statistical Thermodynamics; University Press: Cambridge, UK, 1939.

137. Landsberg, P.T. Foundations of thermodynamics. Rev. Mod. Phys. 1956, 28, 363-392.

138. Landsberg, P.T. A comment on Nernst's theorem. J. Phys A: Math. Gen. 1989, 22, 139-141.

139. Belgiorno, F. Notes on the third law of thermodynamics I. J. Phys A: Math. Gen. 2003, 36, 8165.

140. Belgiorno, F. Notes on the third law of thermodynamics II. J. Phys A: Math. Gen. 2003, 36, 8195.

141. Emch, G.G. Algebraic Methods in Statistical Mechanics and Quantum Field Theory; Wiley Interscience: New York, NY, USA, 1972.

142. Bogoliubov, N.N. On the theory of superfluidity. J. Phys. (USSR) 1947, 11, 23.

143. Dumcke, R. The low density limit for an N-level system interacting with a free Bose or Fermi gas. Commun. Math. Phys. 1985, 97, 331-359.

144. Whitney, R.S. Thermodynamic and quantum bounds on nonlinear dc thermoelectric transport. Phys. Rev. B 2013, 87, 115404.

145. Bruneau, L.; Jakšić, V.; Pillet, C.-A. Landauer-Büttiker formula and Schrödinger conjecture. 2013, arXiv:1201.3190.

146. Kolar, M.; Gelbwaser-Klimovsky, D.; Alicki, R.; Kurizki, G. Quantum bath refrigeration towards absolute zero: Unattainability principle challenged. Phys. Rev. Lett. 2012, 108, 090601.

(c) 2013 by the author; licensee MDPI, Basel, Switzerland. This article is an open access article distributed under the terms and conditions of the Creative Commons Attribution license (http://creativecommons.org/licenses/by/3.0/). 